\title{
The occurrence frequency of auroral potential structures and electric fields as a function of altitude using Polar/EFI data
}

\author{
P. Janhunen ${ }^{1}$, A. Olsson ${ }^{2}$, and H. Laakso ${ }^{3}$ \\ ${ }^{1}$ Finnish Meteorological Institute, Geophysical Research, Helsinki, Finland \\ ${ }^{2}$ Swedish Institute of Space Physics, Uppsala Division, Uppsala, Sweden \\ ${ }^{3}$ ESTEC, Space Science Department, Noordwijk, The Netherlands
}

Received: 15 January 2003 - Revised: 28 August 2003 - Accepted: 4 September 2003 - Published: 2 April 2004

\begin{abstract}
The aim of the paper is to study how auroral potential structures close at high altitude. We analyse all electric field data collected by Polar on auroral field lines in 1996-2001 by integrating the electric field along the spacecraft orbit to obtain the plasma potential, from which we identify potential minima by an automatic method. From these we estimate the associated effective mapped-down electric field $E_{i}$, defined as the depth of the potential minimum divided by its half-width in the ionosphere. Notice that although we use the ionosphere as a reference altitude, the field $E_{i}$ does not actually exist in the ionosphere but is just a convenient computational quantity. We obtain the statistical distribution of $E_{i}$ as a function of altitude, magnetic local time (MLT), $K_{p}$ index and the footpoint solar illumination condition. Surprisingly, we find two classes of electric field structures. The first class consists of the low-altitude potential structures that are presumably associated with inverted$\mathrm{V}$ regions and discrete auroral arcs and their set of associated phenomena. We show that the first class exists only below $\sim 3 R_{E}$ radial distance, and it occurs in all nightside MLT sectors ( $R_{E}=$ Earth radius). The second class exists only above radial distance $R=4 R_{E}$ and almost only in the midnight MLT sector, with a preference for high $K_{p}$ values. Interestingly, in the middle altitudes $\left(R=3-4 R_{E}\right)$ the number of potential minima is small, suggesting that the low and high altitude classes are not simple field-aligned extensions of each other. This is also underlined by the fact that statistically the high altitude structures seem to be substorm-related, while the low altitude structures seem to correspond to stable auroral arcs. The new finding of the existence of the two classes is important for theories of auroral acceleration, since it supports a closed potential structure model for stable arcs, while during substorms, different superposed processes take place that are associated with the disconnected high-altitude electric field structures.
\end{abstract}

Key words. Magnetospheric physics (electric fields; auroral phenomena) - Space plasma physics (electrostatic structures)

Correspondence to: P. Janhunen

(pekka.janhunen@fmi.fi)

\section{Introduction}

Strong, up to several hundred millivolts per meter, perpendicular electric fields exist at around 5000-13 $000 \mathrm{~km}$ altitude in conjunction with optical auroral arcs and inverted- $\mathrm{V}$ electron precipitation (Mozer et al., 1977). Closer examination of the perpendicular electric fields reveals that they often form a convergent electric field signature (electric fields pointing toward each other in close spatial separation). That the pairs of convergent electric fields are typically not caused by temporal variations can be inferred from the fact that upgoing ion beam energies match with the potential $V$ integrated from the boundary of the structure along the satellite orbit, $V=-\int d \mathbf{s} \times \mathbf{E}$ (McFadden et al., 1998). The potentials $V$ obtained in this way also agree with typical inverted- $\mathrm{V}$ electron energies measured at lower altitudes. Thus, it seems that there exists a negative potential structure for each inverted-V event. From the fact that the occurrence frequency of strong convergent electric fields goes down with decreasing altitude, it was concluded that the potential contours must close above the ionosphere, thus forming an upward parallel electric field (Torbert and Mozer, 1978; Mozer et al., 1980; Bennett et al., 1983). This also supports the view that the U-shaped potential model is responsible for generating inverted- $\mathrm{V}$ type electron energy spectra (Carlqvist and Boström, 1970).

The widths of the potential structures should match the typical widths of inverted- $\mathrm{V}$ regions and optical auroral arcs. The widths of inverted- $V$ regions and optical arcs match each other when compared with compatible criteria (StenbaekNielsen et al., 1998; Hallinan et al., 2001). In this paper we consider widths in the range $500 \mathrm{~m}-60 \mathrm{~km}$. This width range should contain almost all auroral arcs, apart from the most narrow ones (Maggs and Davis, 1968; Borovsky, 1993; Knudsen et al., 2001). Knudsen et al. (2001) used the term "mesoscale arc" to refer to what we call "auroral arc" in this paper.

Since there is a lot of support for the U-shaped potential model from low-altitude observations (altitude below $13000 \mathrm{~km}$ ), a natural question arises in what way the U-shaped potential contours continue at higher altitudes. It is topologically necessary that all potential contours close 
somewhere within the flux tube, either below the equatorial plane or in the opposite hemisphere. In this paper, by "lowaltitude" we refer to radial distances below $3 R_{E}$ which corresponds to altitude $13000 \mathrm{~km}$. Surprisingly, no clear evidence of significant convergent electric field signatures was found above about $20000 \mathrm{~km}$ altitude in a statistical study using 78 events for which simultaneous ground magnetometer data were available to rule out substorm cases (Janhunen et al., 1999). Stable arcs are so common phenomena that among 78 events there should be some, thus the indirect implication was that above stable arcs, no clear evidence of significant convergent fields was found.

In this paper we revisit the potential structure closure question in a more thorough and quantitative way by using a much larger data set and complete altitude coverage. Our expanded study is now made possible by the fact that the orbit of Polar has evolved in such a way that no altitude gaps remain within $5000-30000 \mathrm{~km}$. We study potential structures of various depths as a function of altitude, without, however, forgetting magnetic local time (MLT), solar illumination and $K_{p}$ index dependence. In particular, we investigate the so-called effective electric field $E_{i}$, defined as the depth of the potential minimum divided by the width of the structure in the ionosphere. Notice that although we use the ionosphere as a reference altitude, the field $E_{i}$ does not actually exist in the ionosphere but is just a convenient computational quantity. The statistical results can reveal important facts that can be used to constrain various theories that have been put forward to self-consistently explain the yet unsolved electron acceleration mechanism whose manifestations are potential structures, inverted-V regions, ion beams, and auroral arcs (Carlqvist and Boström, 1970; Bryant and Perry, 1995; Janhunen and Olsson, 2000). To complement the study of potential minimum associated electric fields, in Sect. 5 we will also present results concerning the altitude distribution of all perpendicular electric fields (i.e. not only those that are associated with potential minima). That substudy is slightly similar to a statistical study by Lindqvist and Marklund (1990), who used Viking data up to $14000 \mathrm{~km}$ altitude $\left(3.2 R_{E}\right.$ radial distance).

We use the following terminology. "Altitude $(h)$ " means distance from Earth's surface and "radial distance $(R)$ " means distance from the centre of the Earth (geocentric distance). They are connected by $R=R_{E}+h$, where $R_{E}=6371.2 \mathrm{~km}$ is Earth's radius. We usually express altitude in kilometres and radial distance in $R_{E}$. In the figures, radial distance $R$ is used.

\section{Instrumentation}

The Polar Electric Field Investigation (EFI) instrument measures the instantaneous electric field in the satellite spin plane continuously with at least 20 samples per second (Harvey et al., 1995). The measurement is made with two pairs of long wire booms, called 1-2 and 3-4. Also, the component which is parallel to the spin axis is measured with a fixed boom pair (called 5-6), which does not, however, always produce an accurate measurement because of the shortness of the booms. Fortunately, since the spin and orbital planes of the satellite are very nearly equal, the 5-6 component hardly contributes anything to the line integral $V$ of the electric field, $V=-\int d \mathbf{s} \times \mathbf{E}$. Thus, we assumed that the 5-6 component is zero instead of using the measured value in our basic statistical studies. To verify that the results do not depend on this assumption we repeated part of the analysis by using the measured 5-6 component and also, as a third option, the electric field computed from 1-2, 3-4 and the condition $\mathbf{E} \times \mathbf{B}=0$. We found that the potential minima computed by the three different methods gave very nearly the same result.

During intense cold ion outflow events, a wake effect parallel to the magnetic field may develop which may cause an offset of $\sim 5 \mathrm{mV} / \mathrm{m}$ in the parallel component if the magnetic field is close to the spin plane, as it is in many of our events. A manual inspection of some of our events showed, however, that this effect does not play a role in our data set. One reason is that we only use the component of the electric field which is parallel to the spacecraft velocity vector, which is usually approximately perpendicular in the auroral crossings. We also use the Magnetic Field Experiment (MFE) on board Polar (Russell et al., 1995) to reject events where the satellite moves too much parallel to the magnetic field.

\section{Data processing}

\subsection{Data selection}

Only the invariant latitude (ILAT) range $64^{\circ}-75^{\circ}$ is included in the analysis. As ILAT values we use the dipolar ILAT parameter values found in standard Polar orbital CDF files. Furthermore, radial distances only up to $R=6 R_{E}$ are considered because at higher radial distances the satellite travel times over the auroral zone become inconveniently long. Only complete auroral crossings are included, i.e. ones in which the ILAT of Polar changes monotonically between $64^{\circ}$ and $75^{\circ}$. Likewise, if any part of the auroral crossing as determined from ILAT has the radial distance beyond $6 R_{E}$, the crossing is not included in the database. Eclipse periods where the satellite is in the optical shadow of the Earth are removed because the DC electric field double probe measurements may not be reliable. Also, periods when the Plasma Source Instrument (PSI) (Moore et al., 1995) is operating are removed.

\subsection{How potential minima are found and processed}

The data processing starts from the plasma potential as a function of time, i.e. the potential $V(t)$ integrated along the spacecraft orbit using the spin plane electric field, and has the following steps:

1. The potential $V$ is interpolated to $0.005^{\circ}$ ILAT grid spacing, which corresponds to about $500 \mathrm{~m}$ resolution in the ionosphere. The temporal resolution of 20 samples 
per second would enable even somewhat higher ILAT resolution, but the chosen resolution was considered sufficient for this study. Based on Knudsen et al. (2001), a mean width of an auroral arc is $18 \pm 9 \mathrm{~km}$ so that $500 \mathrm{~m}$ resolution is high enough.

2. All local minima are found from the ILAT-interpolated potential, i.e. all data points that have a smaller value than their immediate predecessor and successor.

3. A search is initiated left and right from each local minimum, stopping when the values start to decrease again. This produces a tentative interval for the minimum region.

4. The depth of the minimum is defined to be the difference of values at the minimum and at the point (either left or right, whichever yields the smaller depth) where points started to decrease again. This means that some potential minima are possibly underestimated, especially when a minimum occurs at an edge of a larger minimum.

5. A region around the minimum is found which satisfies the condition $V \leq V_{\min }+0.5 V_{\text {depth }}$ where $V_{\min }$ is the potential value at the local minimum and $V_{\text {depth }}$, is the minimum depth defined in the previous item. The width of the minimum is then defined to be the ILAT extent of the region. This corresponds to width at the half maximum.

6. Interpolation of the data to a new ILAT grid having half the previous resolution is carried out, and the above steps are repeated. This is done in order to prevent left/right searches from getting stuck with local fluctuations, i.e. the data vector is analysed in multiple spatial scales in order to find the minima at any scale. This is repeated recursively to higher coarsening levels until the ILAT spacing is large $\left(>0.5^{\circ}\right)$. This is the most important point of our analysis. The procedure is illustrated in Fig. 1. The first six panels show the original data (top panel), together with five subsequent smoothed versions. The potential becomes smoother in each panel as compared to the panel above. The minima found at each stage are shown as dots. The widths and depths of the minima are indicated by horizontal and vertical lines, respectively. The figure also contains two other panels which are discussed below after item 8 .

7. The parameters (location, width, etc.) of all minima are stored in a file.

8. As a postprocessing step, minima that are too close to each other are replaced by one (the one with the largest depth is selected among equivalent ones). Two minima (belonging to the same auroral pass) are considered equivalent, if and only if all the following conditions hold:

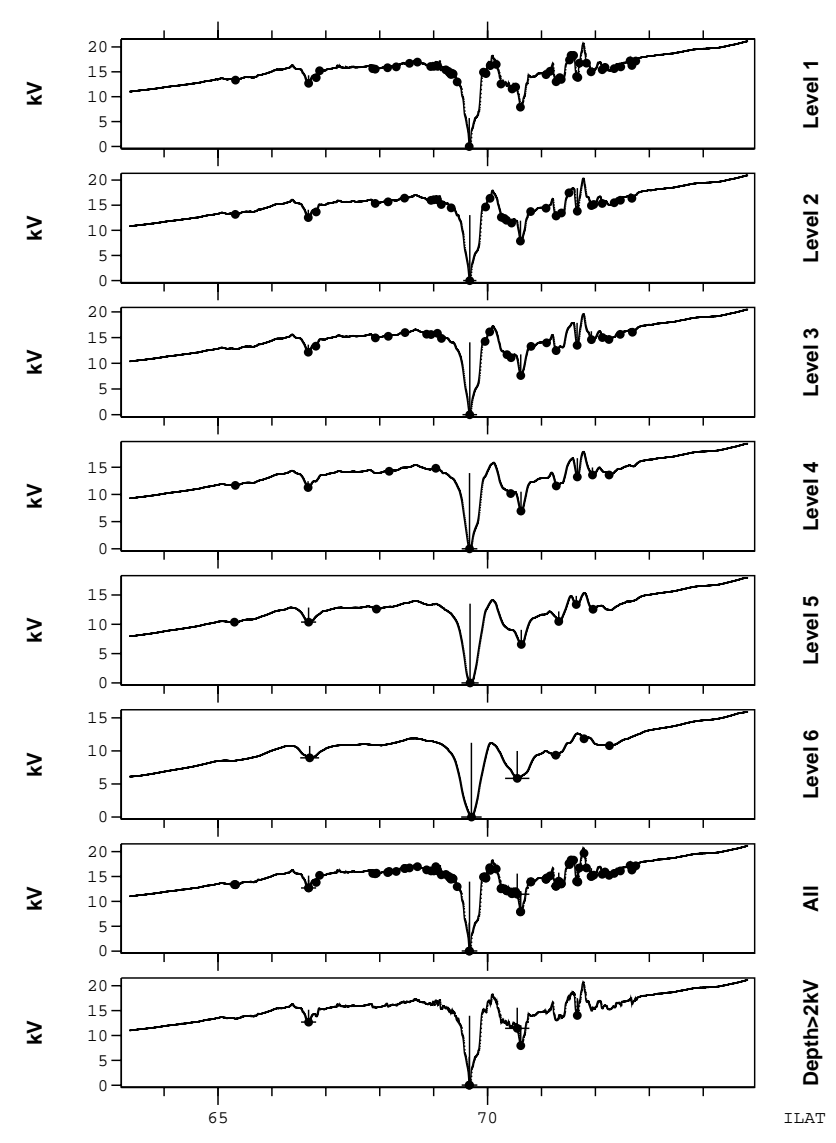

Fig. 1. Illustration of the potential minimum finding algorithm. Panels 1-6 show the first six coarsening levels. Dots show each located local minimum and its width and depth by horizontal and vertical lines, respectively. Panel 7 shows all potential minima after postprocessing (equivalents removed and replaced by the deepest minimum). Panel 8 is same as panel 7, except showing only potential minima deeper than $2 \mathrm{kV}$. In the actual analysis the limit is set to $0.5 \mathrm{kV}$.

(a) The minima come from different coarsening levels;

(b) Their ILAT positions are closer to each other than $(1 / 3) \min \left(W_{1}, W_{2}\right)$, where $W_{1}$ and $W_{2}$ are the ILAT widths of the two minima, respectively;

(c) The widths do not differ too much, for example, $1 / W_{0}<W_{1} / W_{2}<W_{0}$ must hold where $W_{0}=1.5$.

Panel 7 of Fig. 1 repeats the original, unsmoothed potential with the minima found at all smoothing levels, but with "equivalent" minima removed by the algorithm described above in item 8 . Finally, panel 8 shows which of the minima shown in panel 7 remain when only minima deeper than $2 \mathrm{kV}$ are retained. By visual judgement, the minima in panel 8 correspond rather nicely to the real potential minima of the original data vector.

Thus, each auroral crossing may, and in general will, yield multiple potential minima. Furthermore, if a minimum is found at one coarsening level, it is typically found at some 


\section{Ground speed of Polar}

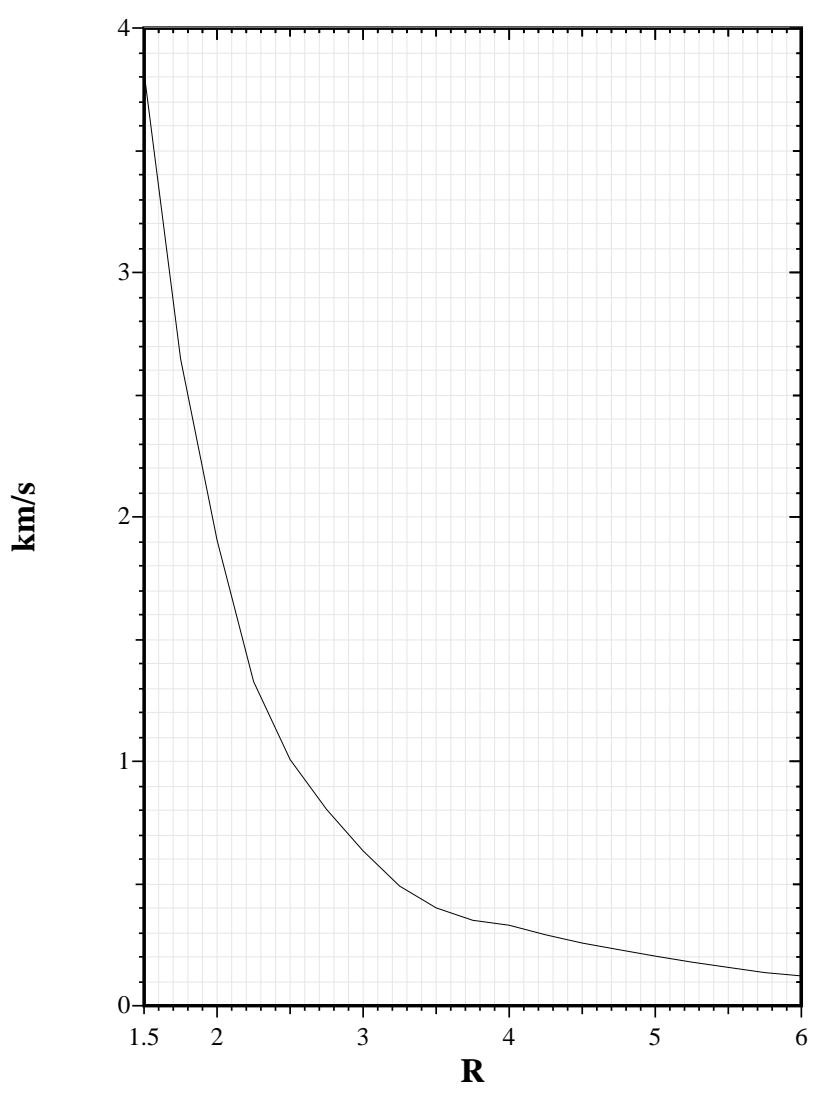

Fig. 2. The ILAT speed of the Polar satellite footpoint in the ionosphere, transformed to $\mathrm{km} / \mathrm{s}$ (solid).

of the higher coarsening levels as well. Usually only one of the multiple found minima survives the postprocessing step, which is what one wants. Naturally, the act of deciding which minima are close enough to be considered equivalent is a subjective one. We think, however, that applying some sort of multiple minima removal method is much better than applying none at all, for example, because in the latter case narrow minima would be found at many coarsening levels and thus be statistically overrepresented. We have also checked that changing the criteria within a reasonable range does not appreciably affect the statistical results.

\subsection{Postprocessing steps}

Before plotting and generating the statistical results presented below, some further simple filtering conditions are applied, which are listed here:

1. The angle between the ionosphere-projected satellite trajectory with constant ILAT circles must be between $60^{\circ}$ and $120^{\circ}$. This is done to remove passes where the satellite moves nearly tangential to the auroral oval because in such geometries, the satellite ILAT speed is low and the geometry is unfavourable for analysing the trajectory-integrated potential.
2. The angle between $\mathbf{B}$ and the satellite velocity vector must be at least $30^{\circ}$, because if the satellite moves almost parallel to the field line, the ILAT speed is again low and temporal variations easily interfere with the analysis.

3. Only events with a depth larger than $0.5 \mathrm{kV}$ are shown, unless otherwise noted. The number of potential minima grows rapidly if one reduces the lower depth threshold.

In order to display statistical results, the orbital coverage is needed as a function of altitude, MLT, $K_{p}$, and season. Since we are expecting to measure static structures that the orbit intersects, it makes more sense to define the orbital coverage as the number of oval crossings rather than as the time spent. As the altitude in general varies somewhat as the satellite moves between $64^{\circ}$ and $75^{\circ}$ ILAT, one has to decide which altitude is defined as the altitude of the oval crossing. Notice that we cannot use the altitude at which the potential minimum is truly observed because there are usually several (or none at all) potential minima per oval crossing and the orbital coverage must be computed in a way which is independent of the occurrence of the minima. We choose to define the altitude of the oval crossing to be the altitude where the satellite was when it crossed the nominal $Q=2$ oval in Table 1 of Holzworth and Meng (1975).

\subsection{Structure lifetime and motion effects}

The ILAT speed of Polar is highly dependent on altitude and is about $0.002^{\circ}$ per s at $R=5 R_{E}$ (Fig. 2). With this speed, it takes about $50 \mathrm{~s}$ to pass through a potential minimum of $0.1^{\circ}$ width. This time is shorter than typical lifetimes of auroral arcs, so the satellite does not miss such structures because of structure lifetime effects. Some of the widest minima considered in this study $\left(0.6^{\circ}\right)$ could be possibly affected at high altitudes, since it takes about $5 \mathrm{~min}$ to traverse through them, but we think that this cannot distort the statistics in a significant way.

The possibility that arc motion could affect the result is now considered. There are three possible cases: 1) The potential structure is moving towards the satellite. In this case the width and depth of the potential structure are underestimated, but their ratio (the effective electric field) is not affected. However, if the depth is underestimated so much that it drops below our selected threshold $(0.5 \mathrm{kV})$, the structure drops out of the database and in this way the effective electric field statistics are also affected. 2) The potential structure moves in the same direction as the satellite with an speed which is lower than the satellite speed. In this case the width and depth of the potential structure are overestimated, but again, the effective electric field is not affected. However, weak potential structures, that in reality are below the threshold, can be raised above the threshold and thus also affect the effective electric field statistics. 3) The potential structure moves in the same direction as the satellite with a speed which is larger than the satellite speed. In this case the 
Occ. freq of $E i>100 \mathrm{mV} / \mathrm{m}, \mathrm{v}=100 \mathrm{~m} / \mathrm{s}$

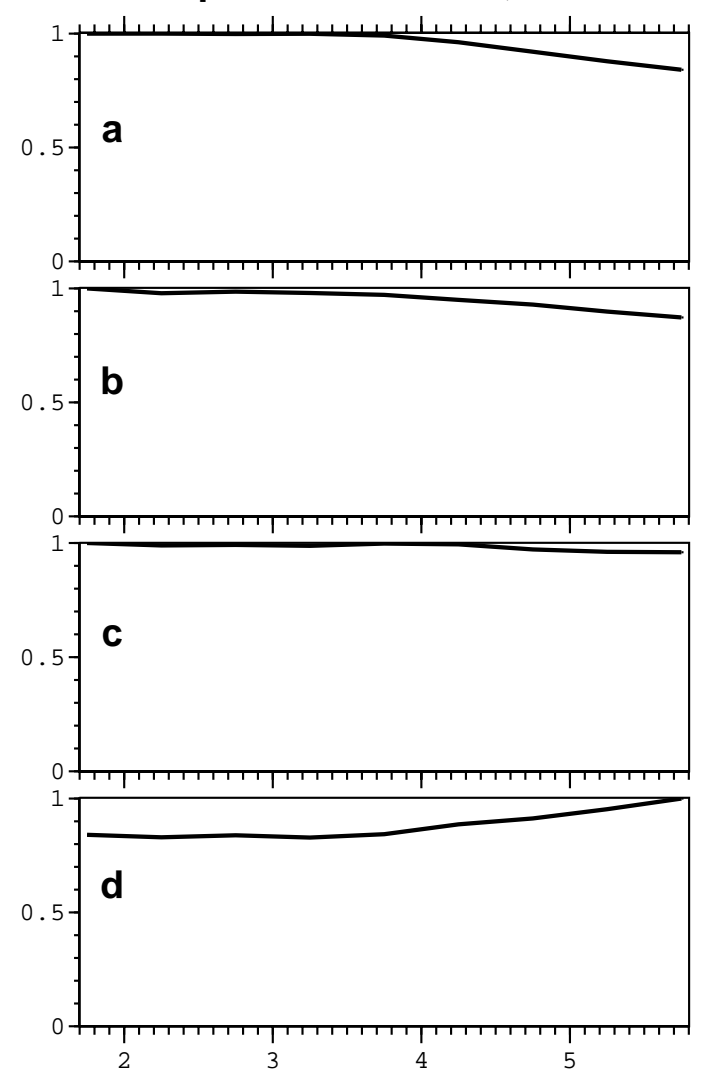

Occ. freq of $E i>100 \mathrm{mV} / \mathrm{m}, \mathrm{v}=200 \mathrm{~m} / \mathrm{s}$

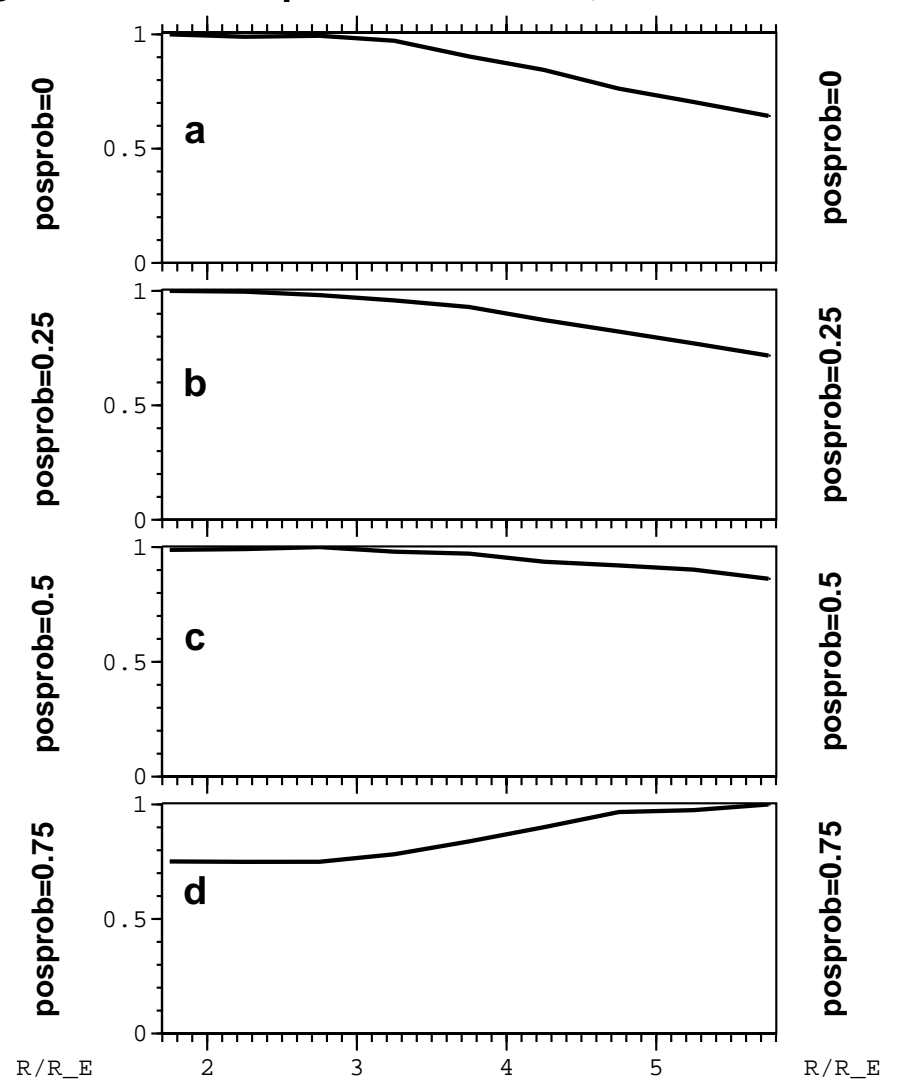

Fig. 3. Simulated effect of structure motion to effective mapped-down electric field occurrence frequency as a function of radial distance, taking into account realistic Polar satellite speed at different altitudes. Panels (a)-(d) correspond to $0 \%, 25 \%, 50 \%$ and $75 \%$, respectively, of the true potential structures being positive (black aurora structures). In the left plot the average structure speed in the ionosphere is $100 \mathrm{~m} / \mathrm{s}$ and in the right plot it is $200 \mathrm{~m} / \mathrm{s}$.

polarity of the potential structure will come out reversed: a negative structure appears as a positive one and vice versa. Whether this causes a net underestimation or overestimation of the occurrence frequency of effective electric fields depends on the relative occurrence frequencies of negative and positive structures, which is unknown.

In summary, for structures that move slower than the satellite, the effects that occur for structures moving opposite or parallel to the satellite, at least qualitatively, tend to cancel each other out. Overall, some underestimation of the potential structure occurrence frequency takes place if the structures are fast-moving; exactly how much depends on how many positive potential structures there are. Concerning the effective electric field distribution of detected potential structures, their distribution is essentially independent of the velocity of the structures.

To evaluate structure motion effects more quantitatively, we perform the following simple Monte Carlo simulation. As random variables we use (i) the potential structure depth $V$, which is exponentially distributed, (ii) the potential structure ionospheric width, which obeys a uniform distribution between 0 and $60 \mathrm{~km}$, and (iii) the ionospheric veloc- ity of the structure $v$, which is exponentially distributed with $v_{0}=100 \mathrm{~m} / \mathrm{s}$ or $200 \mathrm{~m} / \mathrm{s}$ expectation value and a random sign. The potential depth $V$ is modified by the factor $\left|v_{s}\right| /\left|v-v_{s}\right|$, where $v_{s}$ is the satellite speed (a realistic speed specific to each altitude is employed, Fig. 2) and multiplied by -1 , if $v$ and $v_{s}$ have the same sign and $|v|>\left|v_{s}\right|$.

The results of the simulation are shown in Fig. 3. In the left plot the mean ionospheric speed of the structure is $100 \mathrm{~m} / \mathrm{s}$ and in the right plot it is $200 \mathrm{~m} / \mathrm{s}$. To our knowledge, no large statistical studies of auroral arc speeds exist, but in event studies the speeds are mostly below $200 \mathrm{~m} / \mathrm{s}$, although speeds up to $400-500 \mathrm{~m} / \mathrm{s}$ can also occur (Haerendel et al., 1993; Williams et al., 1998; Trondsen and Cogger, 2001); thus, the values 100 and $200 \mathrm{~m} / \mathrm{s}$ shown in the plot pair of Fig. 3 should be representative. Notice that since the speeds are exponentially distributed, there are also much higher speeds than 200 $\mathrm{m} / \mathrm{s}$ present in the simulated ensemble of potential structures. The quantity plotted in each panel of Fig. 3 is the number of negative potential structures reported by the simulated satellite at each altitude, divided by the true number of them. In panels (a) all the potential structures in the ensemble are assumed negative. At low altitude the satellite correctly detects 


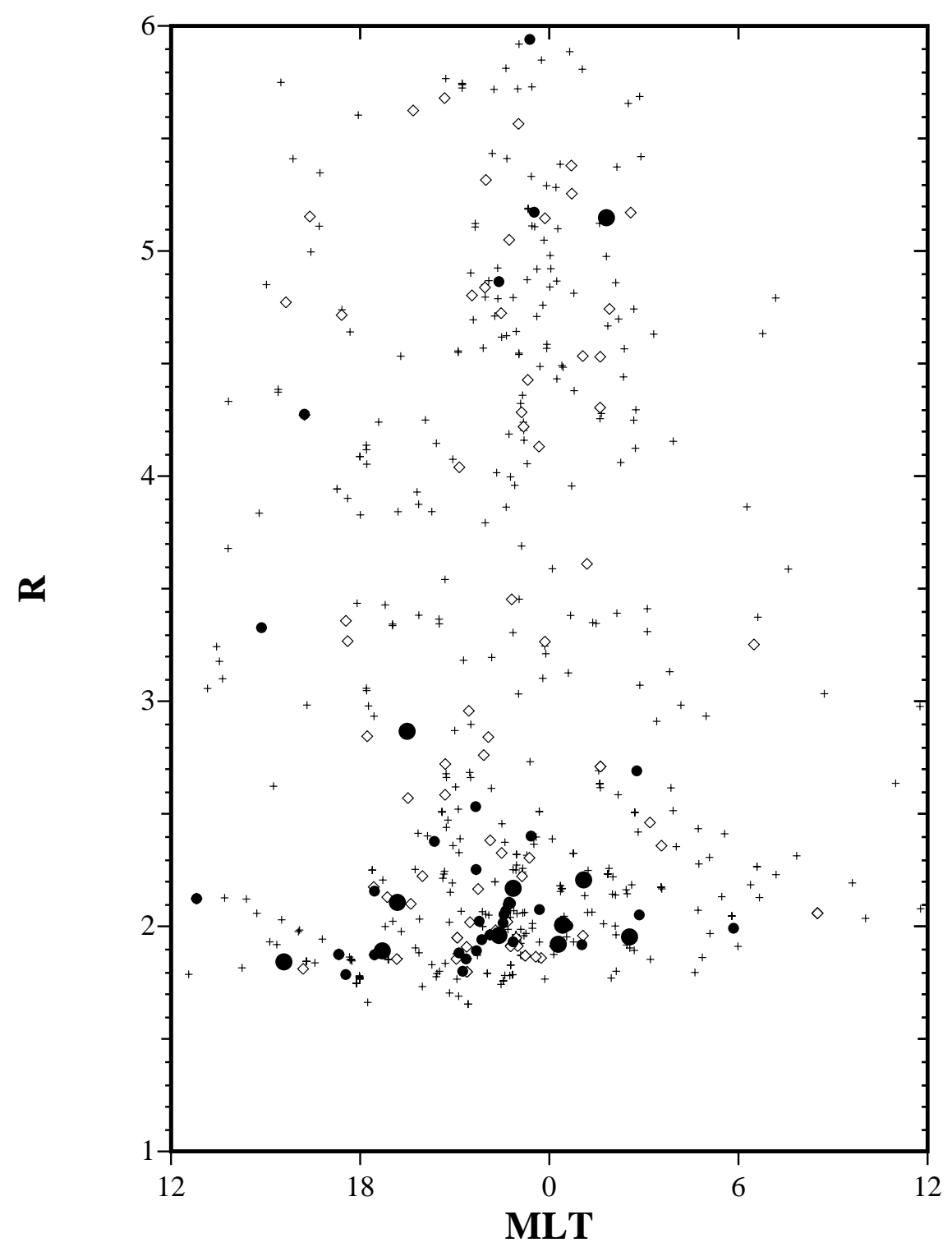

Fig. 4. All potential minima larger than $2 \mathrm{kV}$ and with width $<0.6^{\circ}(60 \mathrm{~km}$ in the ionosphere) as a function of MLT and radial distance $R$. Different energies (minimum depths) are shown by different symbols: $2-4 \mathrm{kV}$ plus sign, 4-6 diamond, 6-8 small black disk, more than 8 large black disk.

all of them (the occurrence frequency is 1), but at high altitude it fails to detect those structures that move in the same direction as the satellite with a higher, speed because it misinterprets them as positive structures. The effect is most pronounced in the right plot because there the structure speed is two times higher, on the average. In panels (b-d), some fraction of the structures in the ensemble are assumed to be positive $(25 \%, 50 \%$ and $75 \%$, respectively). We believe that values around 50\% (panel (c)) are the most likely. Those positive structures that move in the same direction as the satellite with a higher speed are now misinterpreted as negative ones and thus, they increase their reported number, partly or completely balancing the effect. If the number of positive and negative structures is the same (panel (c)), the net influence of the structure speed to the number of detected structures is almost nonexistent. The conclusion that we draw from Fig. 3 is that the structure motion effect on the occurrence frequency of effective electric fields is likely to be negligible below $4 R_{E}$ radial distance and about $10-20 \%$ at $6 R_{E}$. In the worst case $\left(v_{0}=200 \mathrm{~m} / \mathrm{s}\right.$, no positive structures at all), the effect would be about $40 \%$ at $6 R_{E}$ and negligible below $3.5 R_{E}$.

\subsection{Effect of mapping}

As mentioned above, we use the dipole ILAT throughout the paper. The employed ILAT definition has some effect to the structure width in the ionosphere, because the mapping depends on the underlying magnetic field model. To evaluate 


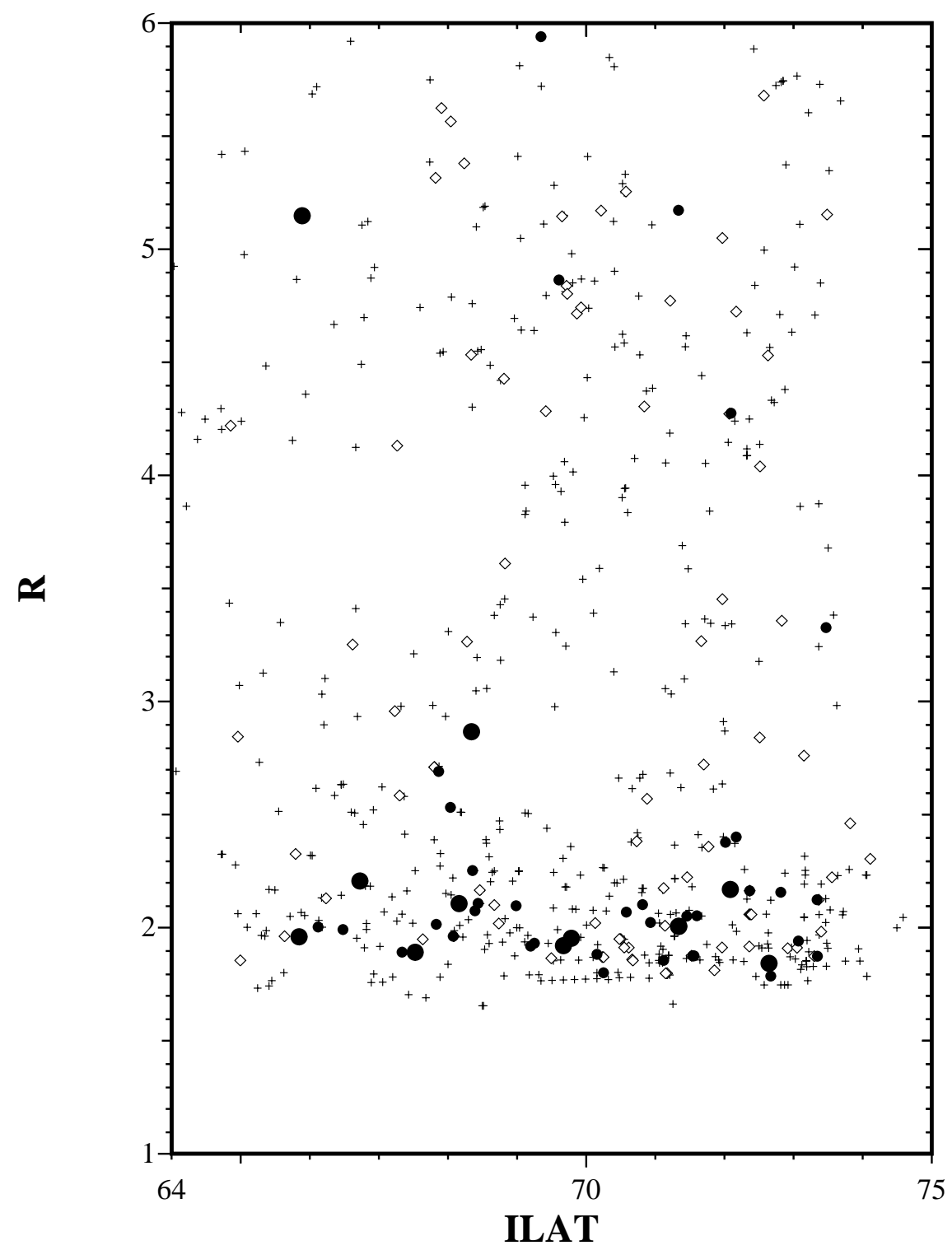

Fig. 5. Distribution of the minima against ILAT and $R$ for the nightside (18:00-06:00 MLT) events. For details of the symbols, see caption of Fig. 4.

the effect quantitatively, we check how much the structure widths would differ if the Tsyganenko-89 magnetic field model, together with IGRF internal field model, are used instead of a dipole model. The result is that in $50 \%$ of the cases, the difference is less than $11 \%$, and in $90 \%$ of the cases it is less than $33 \%$. Almost the same numbers are obtained regardless of which $K_{p}$ value (1-6) one uses in the Tsyganenko model; thus, we conclude that the magnetic field model used to define the ILAT does not have a significant effect on our results.

Another effect of mapping is that ILAT values below 66 map below $R=6 R_{E}$ in the equatorial plane. In other words, in the highest radial distance bin $\left(5.5 R_{E}<R<6 R_{E}\right)$, the lowest ILAT values are not covered by the satellite. In principle, this may distort the statistics in the highest radial distance bin to some extent, although the effect should be rather minor since usually ILAT below 66 represents subauroral latitudes where potential structures do not exist. The conclusions of the paper are not affected since they do not depend on the data in the highest radial distance bin.

\section{Results for potential minima and their associated electric fields}

As an overview, Fig. 4 shows all potential minima deeper than $2 \mathrm{kV}$ as a function of MLT and radial distance $R$. Different symbols indicate the depth of the minima (see figure caption for details). Most potential minima are found at 18:00-04:00 MLT and the highest energy potential minima 

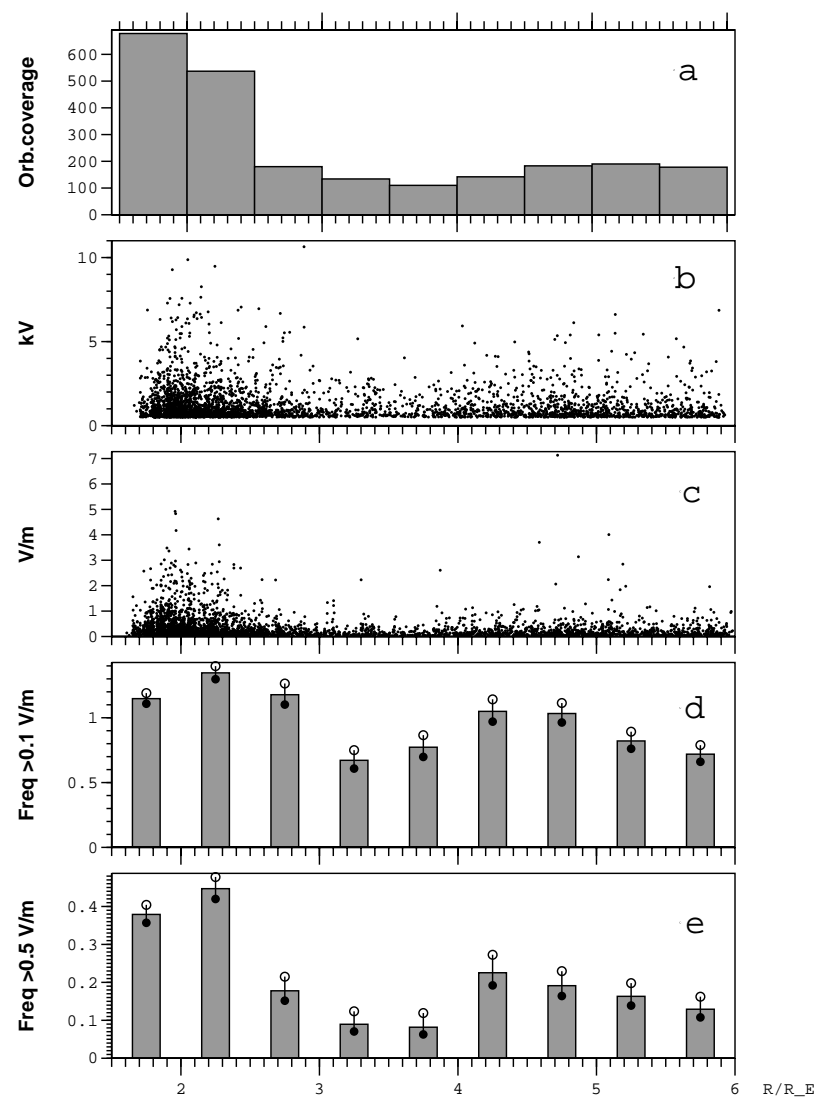

Fig. 6. All nightside potential minima deeper than $0.5 \mathrm{kV}$ and the corresponding effective ionospheric electric fields $E_{i}$ : (a) number of orbital crossings in each radial bin, (b) depth in kilovolts and radial distance of each potential minimum, (c) effective ionospheric electric field associated with each potential minimum with lower limit $100 \mathrm{mV} / \mathrm{m}$, (d) occurrence frequency of $E_{i}$ being larger than $100 \mathrm{mV} / \mathrm{m}$ (number of points in panel (c) divided by panel (a), (e) occurrence frequency of $E_{i}$ being larger than $500 \mathrm{mV} / \mathrm{m}$.

are found at low altitudes, below $3 R_{E}$. Similarly, Fig. 5 displays the minima as a function of ILAT and $R$ for the nightside (18:00-06:00 MLT). The potential minima tend to occur uniformly over the 65-74 ILAT range, partially because no MLT separation was done here (both the intrinsic variability and the dependence of the mean auroral oval on MLT contribute to the spreading of the distribution). Notice that in Figs. 4 and 5, the time spent by the satellite in different regions is not taken into account. In the subsequent plots the orbital coverage will be taken into account.

\subsection{Altitude}

Our baseline plot is Fig. 6 that shows results for all data collected in the nightside including all $K_{p}$ values. Panel (a) is the number of orbital crossings in each $0.5 R_{E}$ radial bin. Panel (b) shows the depth in kilovolts of all found potential minima which are deeper than $500 \mathrm{~V}$ and at most $0.6^{\circ}$ wide in ILAT (corresponding to $60 \mathrm{~km}$ in the ionosphere). Panel (c) shows the effective mapped-down ionospheric elec-

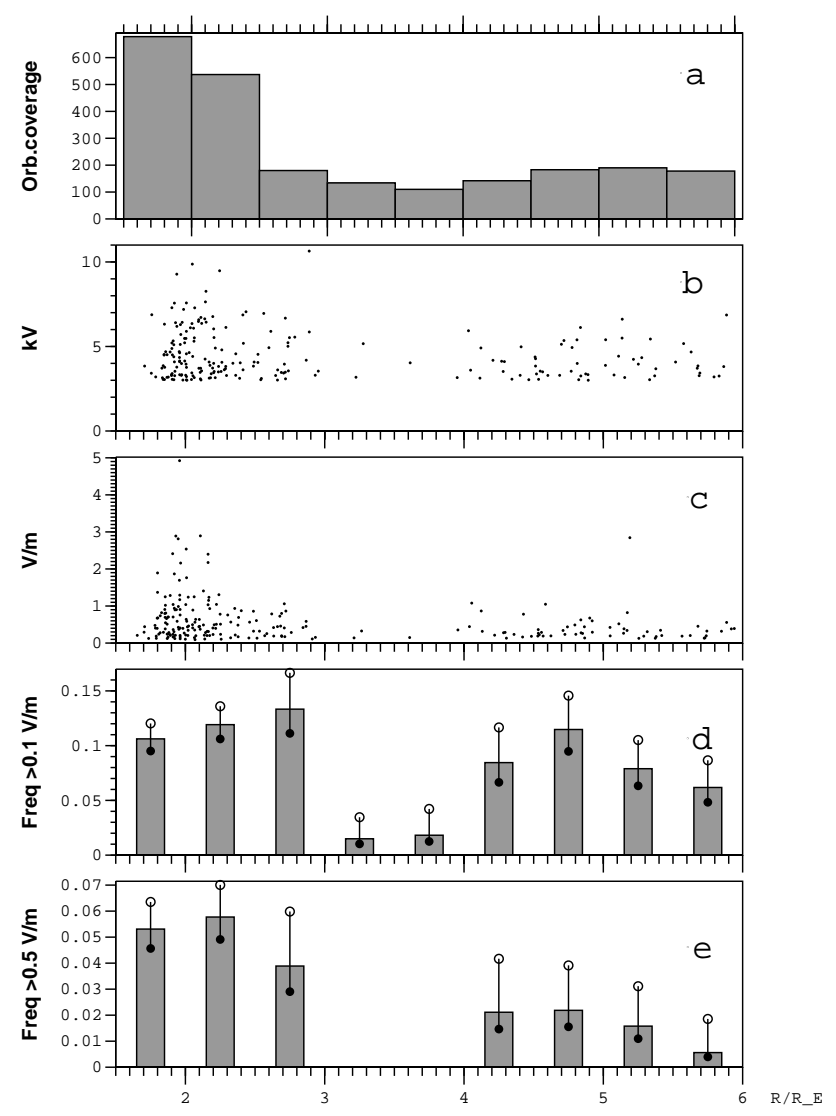

Fig. 7. Same as Fig. 6 but only potential minima deeper than $3 \mathrm{kV}$ are included.

tric field $E_{i}$ in $\mathrm{V} / \mathrm{m}$, associated with all those potential minima plotted in the previous panel that also satisfy the condition $E_{i}>100 \mathrm{mV} / \mathrm{m}$. The effective mapped-down ionospheric electric field is defined as the depth of the potential minimum divided by the mapped-down half-width of the structure in the ionospheric plane. Notice that the effective electric field $E_{i}$ does not really exist in the ionosphere, but we use the ionosphere just as a convenient reference altitude in order to easily compare electric fields measured at different altitudes. This variable is supposed to be invariant with radial distance above the acceleration region, if the acceleration structure is not local but is mapped along magnetic field lines towards the magnetosphere. Panel (d) is the occurrence frequency of $E_{i}>100 \mathrm{mV} / \mathrm{m}$ per orbital crossing, which is obtained by dividing the number of data points in panel (c) in each radial bin by the corresponding orbital crossing number from panel (a) (so " 1 " means that there is one event every orbit, on the average). The error bars correspond to $1 / \sqrt{n}$ relative errors, where $n$ is the number of datapoints exceeding the threshold. Panel (e) is the occurrence frequency of $E_{i}>500 \mathrm{mV} / \mathrm{m}$. Panels (d) and (e) should, in principle, be divided by one of the profiles of simulated $E_{i}$ occurrence frequency displayed in Fig. 3. Applying such a correction would change the results only mildly in the highest radial distance bins, however. 

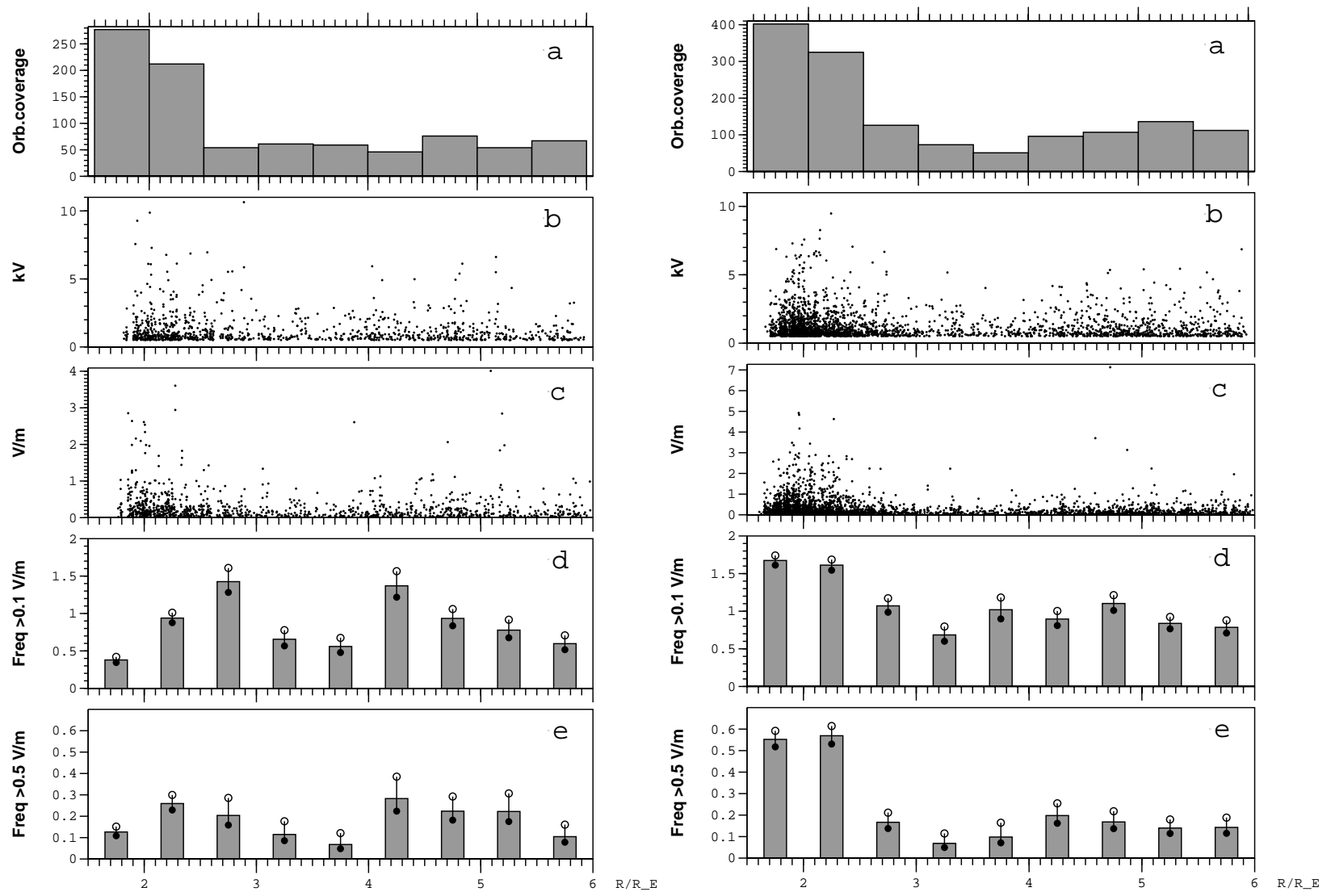

Fig. 8. Same as Fig. 6, but only when the ionosphere at satellite footpoint is sunlit (left plot) and in darkness (right plot).

We see from Fig. 6 (panels (d) and (e)) that the large effective electric fields associated with potential minima occur more frequently at low altitudes than at higher altitudes. The effect is quite pronounced in panel (e), i.e. when the threshold is $500 \mathrm{mV} / \mathrm{m}$. A similar trend can also be seen in the scatter plot of the potential minima (panel (b)). We will see below (Sect. 4.3) that the increase in the occurrence frequency above $4 R_{E}$ is mainly due to phenomena in the midnight MLT sector. Interestingly, an occurrence minimum appears at $3-4 R_{E}$ radial distance. Even though the figure contains all nightside MLT and $K_{p}$ values, this tends to suggest that a simple mapping of the potential upward does not happen in the auroral zone.

In Fig. 7 we show the statistics of only those potential minima that are deeper than $3 \mathrm{kV}$. The trends seen in the baseline plot (Fig. 6) appear in Fig. 7 in a more dramatic form. Now we can distinguish two separate populations of potential minima, and no events occur at 3-4 $R_{E}$ radial distance in the bottom panel, with only a few events in the 2 nd panel from the bottom (see also the scatter plots).

\subsection{Solar illumination dependence}

In Fig. 8 we show the statistics separately for events when the ionosphere at the satellite footpoint is sunlit or in dark- ness. During sunlit conditions (left panels), the maximum of the occurrence frequency of high potential minimum associated $E_{i}$ fields (panel (d)) is shifted to a higher radial distance ( $R=2.75 R_{E}$, corresponding to $11000 \mathrm{~km}$ altitude from Earth's surface) than during darkness $\left(R=1.75-2.25 R_{E}\right.$, the corresponding altitude is $5000-8000 \mathrm{~km}$ ). Notice that we use numbers like 2.75 to uniquely label our bins, not to imply that the precision in altitude is two decimal digits. Furthermore, in both sunlit and darkness cases separately, the maximum occurrence frequency moves towards lower altitudes when the threshold is increased from $100 \mathrm{mV} / \mathrm{m}$ (panel (d)) to $500 \mathrm{mV} / \mathrm{m}$ (panel (e)). This means that strong $E_{i}$ field events tend to occur at lower altitudes. The overall occurrence frequency is quite similar between sunlit and darkness for $>100 \mathrm{mV} / \mathrm{m}$ events, but the low-altitude strong events (panel (e)) are clearly more common in shadow conditions; this may suggest that they tend to occur near local midnight, where the ionospheric footpoint is almost always in darkness. These results are in accordance with recent hybrid simulations of auroral potential structures (Janhunen and Olsson, 2002, Table 2). Both Fig. 8 and the simulations are consistent with the idea that sunlight increases the plasma density at low altitudes due to increased ionospheric photoionisation, which makes the bottom of the acceleration region move upward. This idea was first suggested by Bennett et al. (1983) 

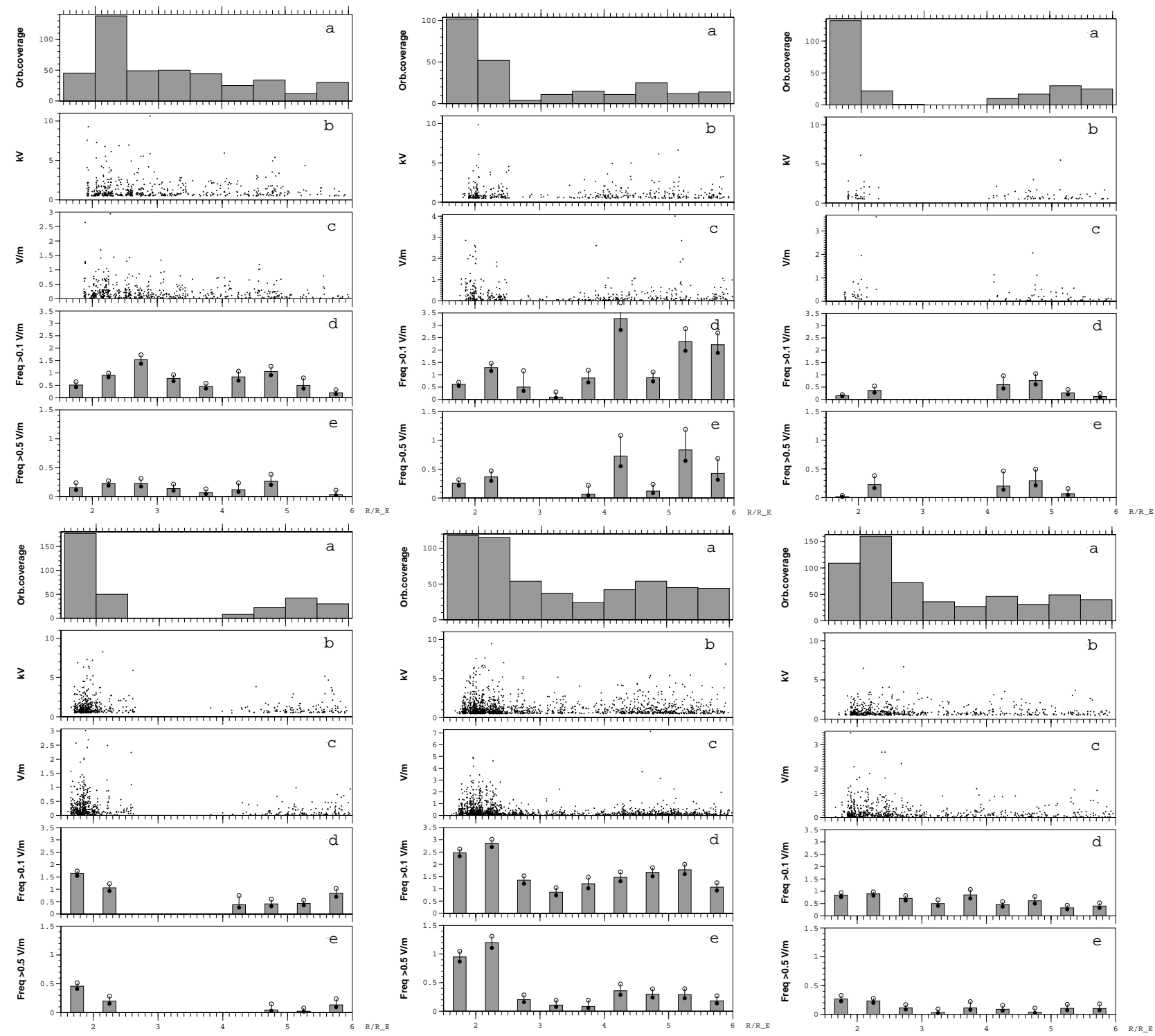

Fig. 9. Same as Fig. 6, but separated in dusk (18:00-22:00), midnight (22:00-02:00) and dawn (02:00-06:00) magnetic local time (MLT) bins, as well as sunlit (top row) and darkness (bottom row) conditions. As in Fig. 6, all $K_{p}$ values are included, width is restricted to be smaller than $0.6^{\circ}$ and depth more than $0.5 \mathrm{kV}$.

based on S3-3 data. Figure 8 suggests that not only the acceleration region bottom altitude moves up, but also potential minima of given depth are rarer during sunlit conditions. In a particular model calculation of the hybrid simulation it was found that halving the ionospheric plasma density changed the potential depth from $3 \mathrm{kV}$ to $3.7 \mathrm{kV}$, and the bottom altitude moved down from $5500 \mathrm{~km}$ to $2600 \mathrm{~km}$ (Janhunen and Olsson (2002), Table 2). Observationally it has also been found using satellite particle data that sunlight suppresses auroral acceleration events (Newell et al., 1996).

\subsection{MLT}

From Fig. 4 it was seen that the potential minima are mainly found in the evening and midnight sectors. To study the MLT distribution in more detail, Fig. 9 shows the statistics separated in three nightside MLT bins (18:00-22:00, 22:0002:00, 02:00-06:00) and separated according to the footpoint illumination conditions.

For the lowest two or three altitude bins we have a reasonable orbital coverage in all six subplots (see the top panels in all subplots) and the behaviour can be summarised as follows. In sunlit conditions, the occurrence frequency is about two times higher in the evening and midnight sectors than 

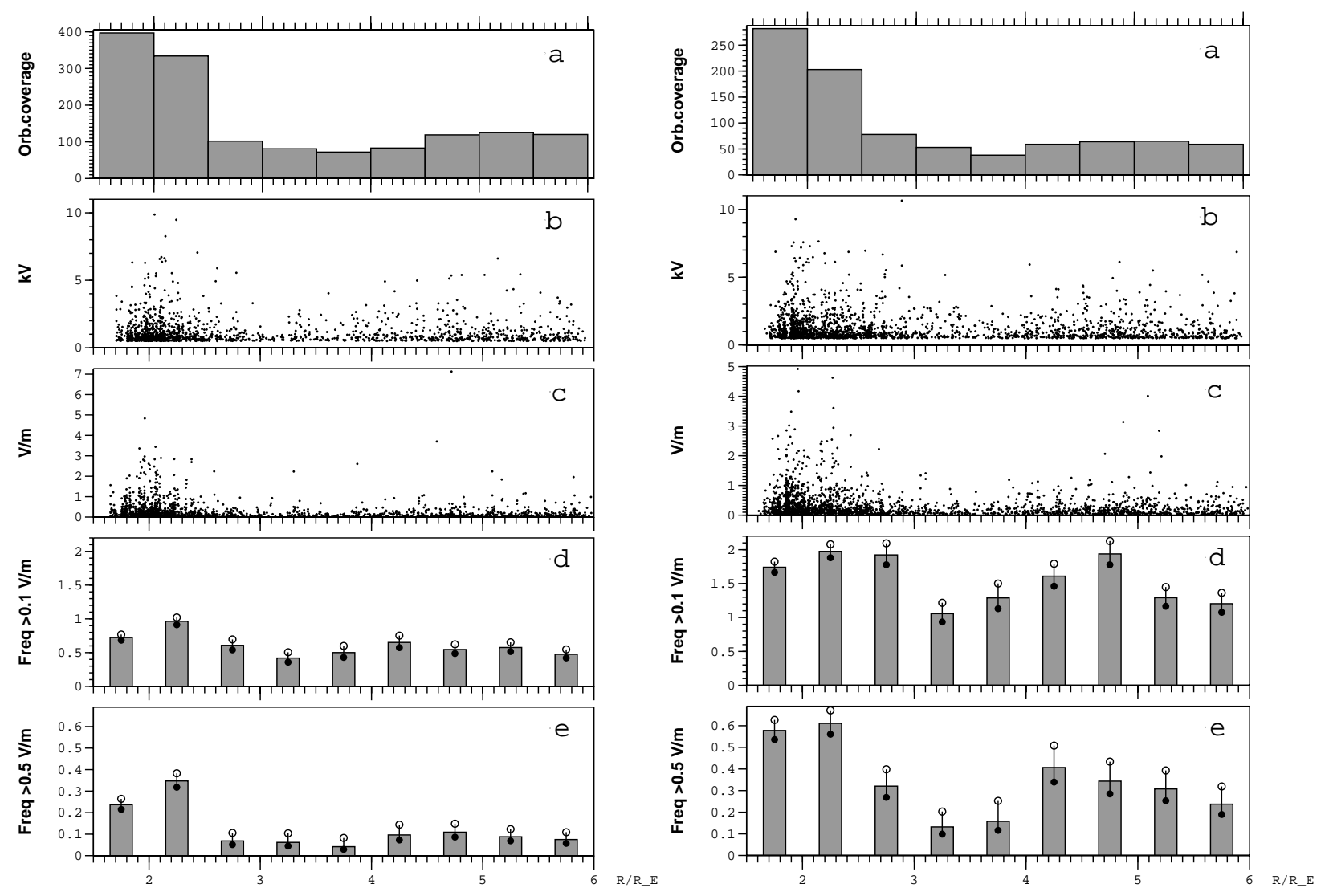

Fig. 10. Same as Fig. 6, but decomposed into small $K_{p}\left(K_{p} \leq 2\right.$, left plot $)$ and large $K_{p}\left(K_{p}>2\right.$, right plot $)$.

in the morning sector. In darkness conditions the highest occurrence frequency is found in the midnight sector, the occurrence frequencies are lower by a factor of 2-3 in the evening sector and the lowest frequencies appear again in the morning sector. These findings are in agreement with earlier studies concerning the occurrence of inverted- $\mathrm{V}$ events at low altitude (Lin and Hoffman, 1982). Overall, the occurrence frequencies in darkness conditions are higher than in sunlit conditions.

For the high altitude bins $\left(R>4 R_{E}\right)$, the data coverage is enough to draw some conclusions. The occurrence frequencies are highest in the midnight sector, both in sunlit and darkness conditions. Instead, in the evening and morning sectors the occurrence frequencies are about equal, which is not the case in the low-altitude events.

Interestingly, the middle altitude occurrence frequencies $\left(R=3-4 R_{E}\right)$ are smaller than the low and high altitude ones in all cases where we have enough data to judge. Such a judgement can be done in the midnight sector for both sunlit and darkness cases, as well as in the evening sector sunlit case and the morning sector darkness case. Such a feature tends to suggest that the low-altitude and high-altitude structures are not necessarily connected.

\section{$4.4 \quad K_{p}$}

$K_{p}$-decomposed statistics are shown in Fig. 10. For the lowaltitude events the occurrence frequency is about two times larger for $K_{p}>2$ than for $K_{p} \leq 2$; also, a bin at $2.75 R_{E}$ contains many events during high $K_{p}$. So the spatial length of the low-altitude potential minimum region may increase with increasing $K_{p}$. For the high-altitude events, the occurrence frequency increases with increasing $K_{p}$; it is 3-4 times larger for $K_{p}>2$ than for $K_{p} \leq 2$. Again, one should notice the existence of a minimum in occurrence rates at 3-4 $R_{E}$ radial distance.

\section{All electric fields}

In the previous section we investigated how often potential minima occur in different regions. As the main measure, we used the effective mapped-down electric field associated with the potential minimum, where the effective electric field $E_{i}$ was defined as the potential minimum depth divided by the mapped-down ionospheric half-width of the structure. To complement this, we now consider all perpendicular electric fields, not only those that are associated with potential minima. We still use the mapped-down version of the measured electric field to be able to easily compare different altitude observations. 

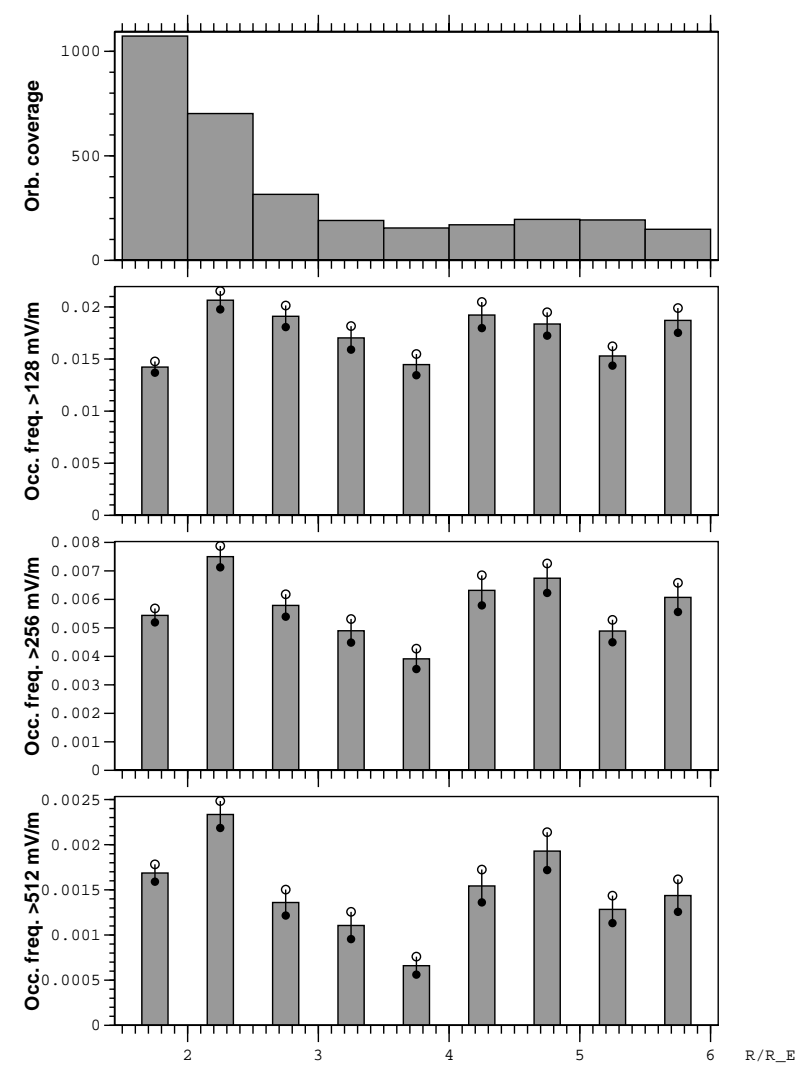

Fig. 11. Baseline plot of altitude distribution of mapped-down perpendicular electric field amplitudes $E_{i}$, including all fields not only those associated with potential minima: (a) number of orbital crossings in each radial bin, (b) occurrence frequency of $E_{i}$ exceeding $100 \mathrm{mV} / \mathrm{m}$, (c) occurrence frequency of $E_{i}$ exceeding $500 \mathrm{mV} / \mathrm{m}$.

\subsection{Data processing}

We take the measured electric field along the spacecraft trajectory and interpolate it to a fixed ILAT grid which has $\sim 10 \mathrm{~m}$ resolution in the ionosphere. Each auroral crossing corresponds to $2^{17}$ data points. Using this high ILAT resolution ensures that we do not undersample the measured field, even at high altitude. The electric field is then multiplied by the ratio of the local satellite displacement divided by the displacement of the satellite footpoint in the ionosphere, to obtain the mapped-down electric field $E_{i}$. Finally, $E_{i}$ is high-pass filtered so that ionospheric scale sizes above $60 \mathrm{~km}$ are removed. The filtering is done for consistency with the previous section results where our largest allowed size for potential minima was $60 \mathrm{~km}$. The selection of data obeys the same rules as those described above for the potential minima, except that one need not remove the orbits quasi-parallel to the magnetic field.

\subsection{Altitude}

In Fig. 11 we show the baseline plot of the electric field altitude distribution, which contains all nightside MLT-sectors and all $K_{p}$ values and illumination conditions. The top panel is the number of orbital crossings. The second, third and fourth panels are the fraction of time (the occurrence frequency, where "1" means that $E_{i}$ is always larger than the given threshold) when $E_{i}$ exceeds 128,256 and $512 \mathrm{mV} / \mathrm{m}$, respectively. Notice that the occurrence frequencies are now numerically much smaller than in the previous section, because in that section the occurrence frequency was defined as the number per orbital crossings, not as a fraction of time, as defined here. We see that large $E_{i}$ fields peak at low altitudes, and there is a minimum at $3.75 R_{E}$. At higher altitudes the fields again become stronger. These trends become clearer as the threshold is increased: at $128 \mathrm{mV} / \mathrm{m}$ threshold the distribution is still almost flat, but at $512 \mathrm{mV} / \mathrm{m}$ threshold the trends are clear. Both trends are similar to what was observed for the potential minimum associated $E_{i}$ fields (Fig. 6). This suggests that potential minimum associated electric fields play an important role among all electric fields in the auroral zone. The error bars correspond to $1 / \sqrt{K}$ relative errors, where $K$ is the number of different orbital crossings having electric fields larger than the threshold. This corresponds to assuming that all datapoints within one crossing are correlated, which need not be true, thus the error bars displayed should be considered as upper limits of the error.

\subsection{Solar illumination}

The sunlit/darkness decomposed statistics are shown in Fig. 12. We see that at low altitude the occurrence frequencies are about two times higher (at $512 \mathrm{mV} / \mathrm{m}$ ) in darkness than in sunlit conditions. At high altitude there is not so much difference. A radial shift of about one bin $\left(0.5 R_{E}\right)$ can be seen in the low-altitude electric fields between sunlit and darkness. Again, the occurrence rates of all electric fields have surprisingly similar profiles as those of the effective electric fields related to potential minima in Fig. 8.

\section{$5.4 \quad$ MLT}

In Fig. 13 we decompose the $E_{i}$ statistics in three MLT sectors, as well as sunlit and darkness conditions. The highaltitude fields that occur in the baseline plot (Fig. 11) appear to come mainly from the midnight MLT sector. In all those MLT/illumination combination plots where we have enough orbital coverage to make a judgement (evening sunlit, midnight sunlit, midnight darkness and morning darkness), there is a minimum in the occurrence frequency of strong mappeddown electric fields around $R=3.5 R_{E}$. Overall, the occurrence frequencies are largest in midnight and smallest in morning. A more detailed look reveals that interestingly, at low altitude the large electric fields are more common in the evening sector than in the morning sector, whereas at high altitude they are equally common in the evening and morning sectors.

\section{$5.5 K_{p}$}

A comparison of low and high $K_{p}$ values is shown in Fig. 14 . The occurrence frequencies of low-altitude electric fields are 

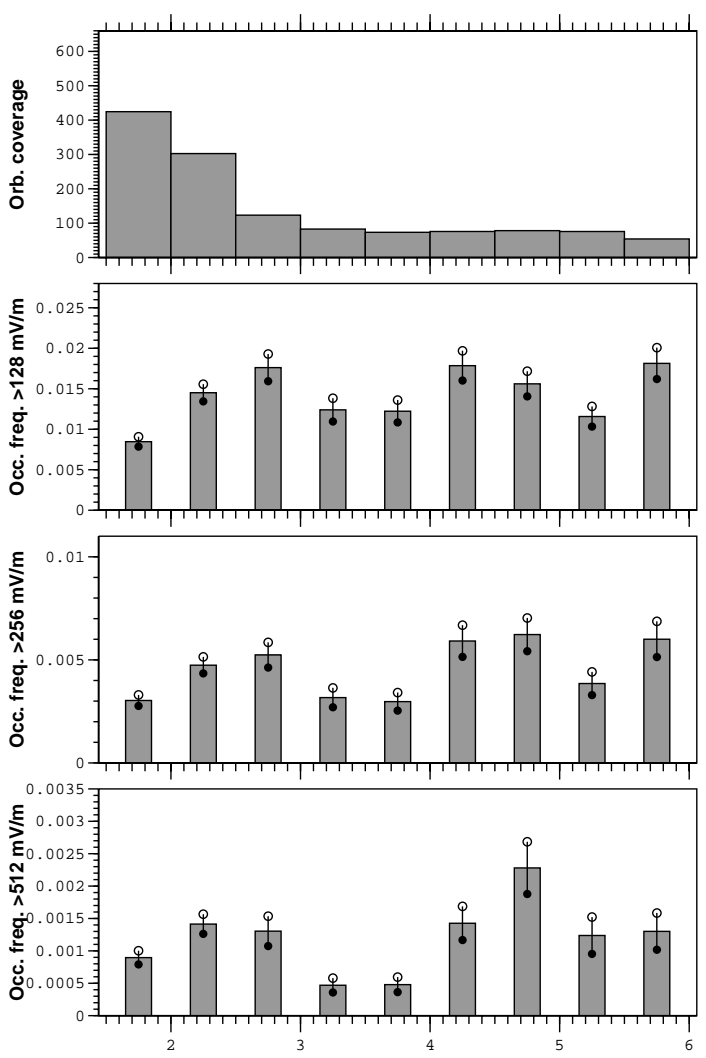
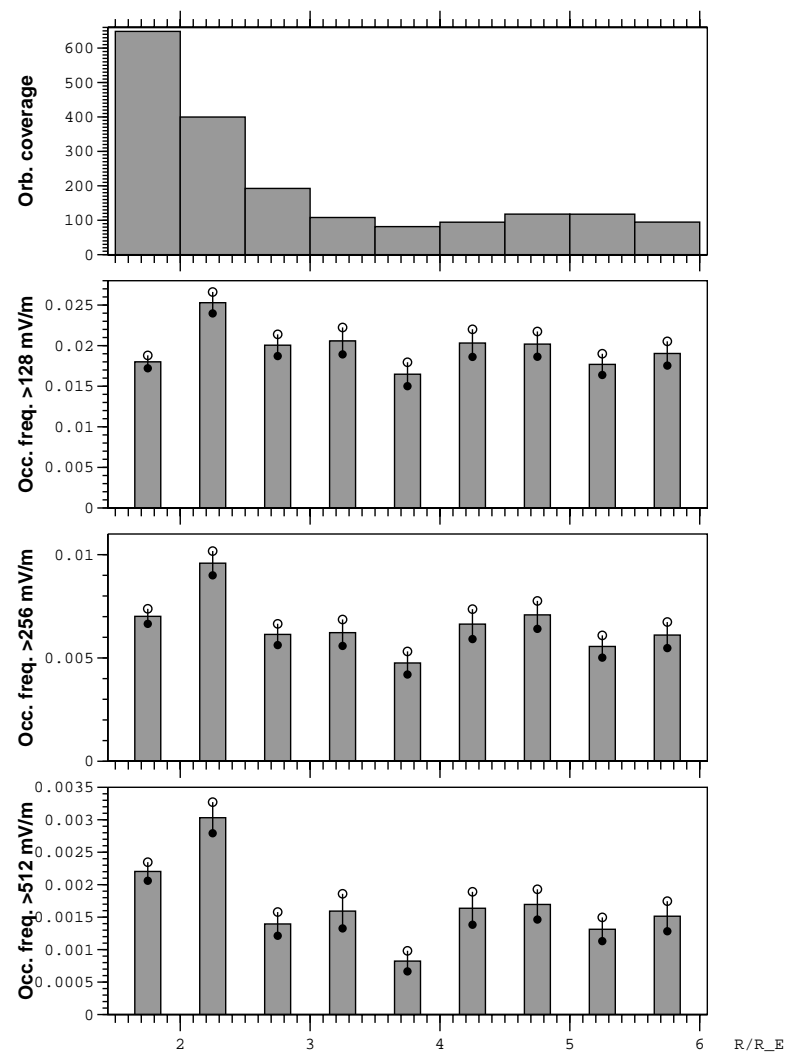

Fig. 12. Same as Fig. 11, but decomposed into sunlit ionospheric footpoint conditions (left) and darkness conditions (right).

about two times larger for high $K_{p}$ than for low $K_{p}$. For high-altitude electric fields, the transition from low to high $K_{p}$ brings about an even larger (about threefold) increase in occurrence frequency, which is similar to the electric fields in the potential minima.

\subsection{Scale size}

In Fig. 15 we show the midnight darkness sector statistics for the effective electric field as a function of the mappeddown scale size (vertical axis). The scale size information is obtained by applying a running average with successively increasing window size and finding at each smoothing level how often the coarse-grained electric field exceeds the given threshold. Superimposed to the plots we also display two curves that correspond to the sampling rate of the instrument (20 samples per $\mathrm{s}$ in normal mode) and the spin frequency. The spin line has no significance, except that some spin contamination might be expected close to it or its harmonic multiples, which we do not see here; however we propose that Fig. 15 shows three categories of electric fields: (1) background electric fields that map along field lines, (2) electric fields of the arc-associated potential structures and (3) other electric fields whose spatio-temporal nature is uncertain. We propose that these categories manifest themselves in the following way in Fig. 15. Background electric fields dominate the top panel, as is natural, since fields of $\sim 100 \mathrm{mV} / \mathrm{m}$ now and then occur in the ionosphere and they vary slowly (i.e. above spin resolution). Arc-associated potential structure electric fields are mainly seen as an island in the $2-2.5 R_{E}$ radial bin and above $\sim 1 \mathrm{~km}$ scale size. They are present in all panels, but one can distinguish them from the background fields most easily in the middle and bottom panels. Everything else seen in the plots is in the third category. At least part of these "other" electric fields are almost certainly associated with waves. Among the "other" electric fields are the high-altitude midnight sector fields mentioned several times earlier in this paper. They tend to peak, for example in 4$5 R_{E}$ bins in the bottom panel.

\subsection{Orbital coverage}

We have identified two clear populations of large electric fields at $\sim 2 R_{E}$ and $4-5 R_{E}$. We have considered possible nongeophysical explanations and found two candidates that could cause the observed middle-altitude minimum in the occurrence frequency. However, as we show below, they are not consistent with our data:

(1) The minimum could be caused by the satellite passing through the altitude bin mostly in unfavourable ILAT. To check this, we plot in Fig. 16 the orbital coverage of Polar as a function of ILAT separately for all MLT sectors and for three radial bins $\left(2-3 R_{E}, 3-4 R_{E}\right.$ and $4-5 R_{E}$, respectively). Only those passages satisfying the trajectory 

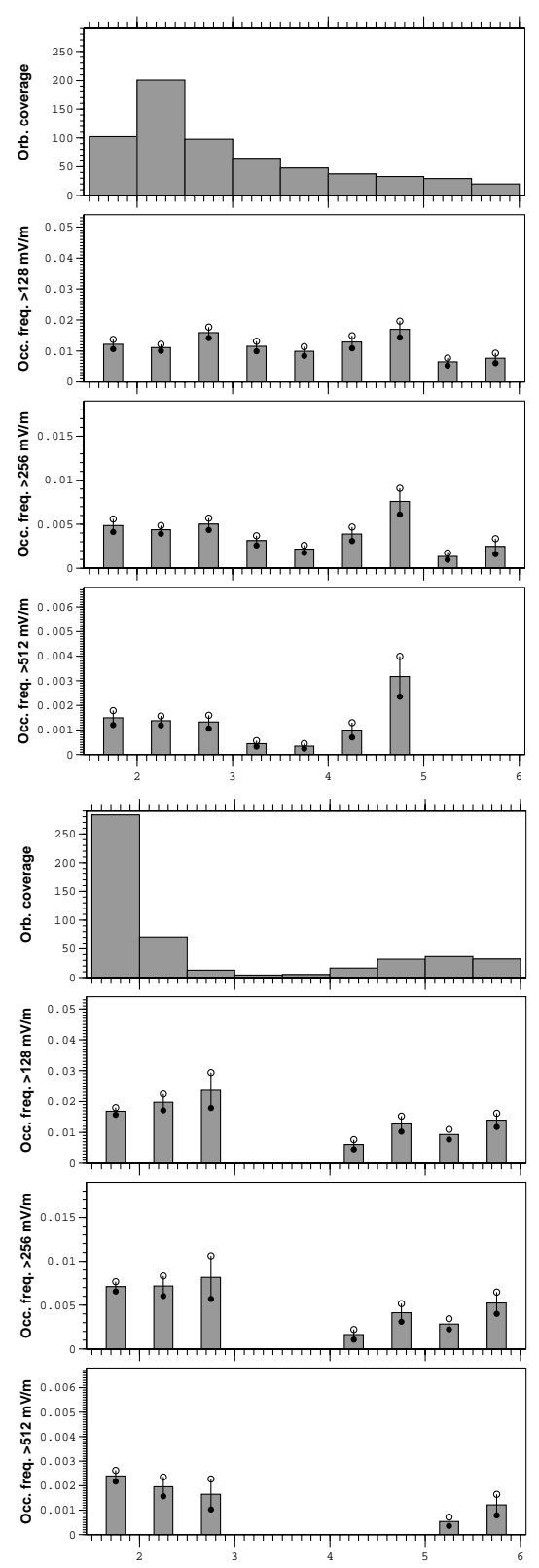
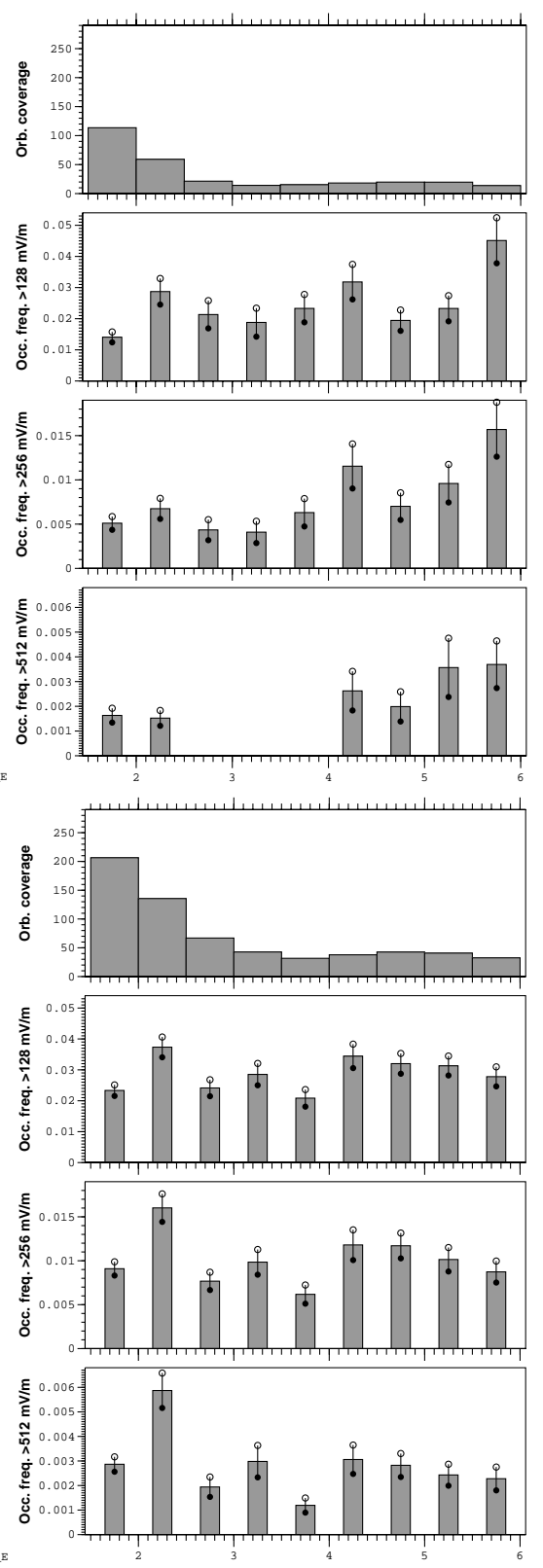
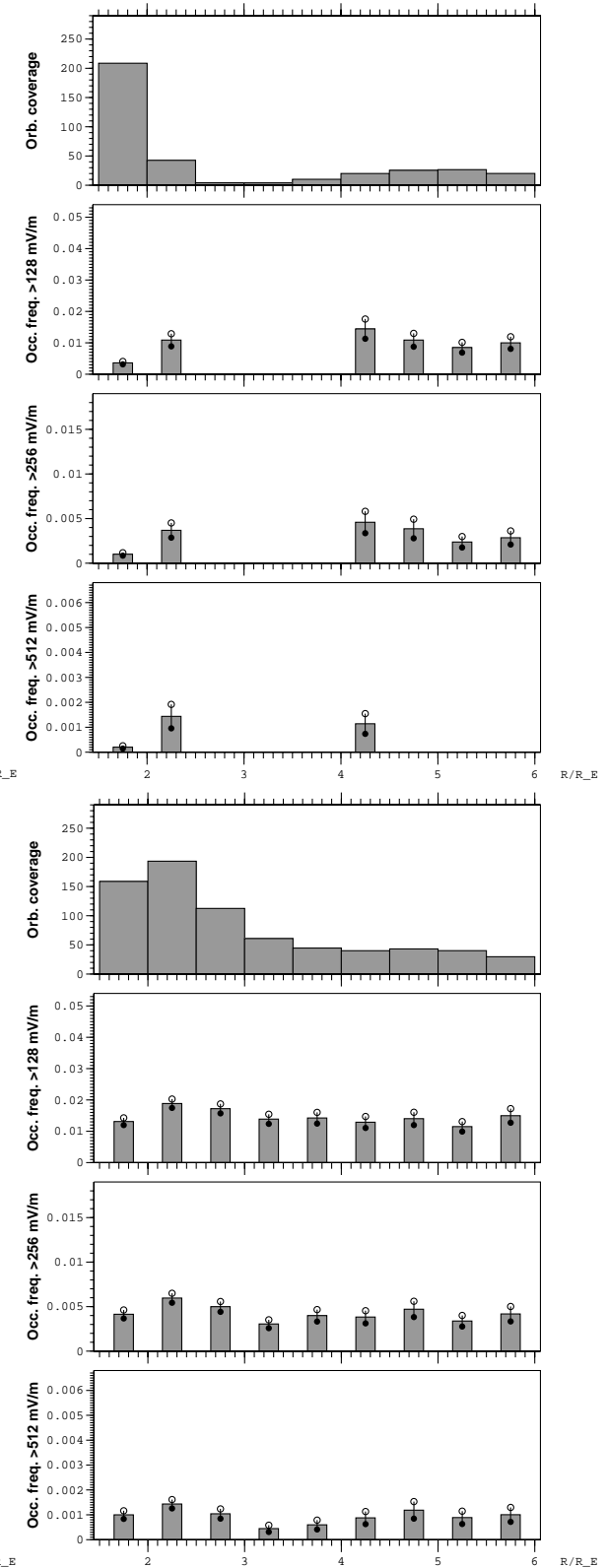

Fig. 13. Same as Fig. 11, but decomposed into different MLT sectors (columns), as well as sunlit (top) and darkness (bottom) conditions.

perpendicularity requirements listed in Sect. 3.2 are included in Fig. 16. The orbital coverage decreases as a function of ILAT at all altitude bins and in all MLT sectors in a rather uniform way. No anomalously small coverage exists in the 3-4 $R_{E}$ radial bin in the most probable auroral oval latitudes as compared to the surrounding radial bins. The orbital coverage in Fig. 16 is plotted as the number of times that each $0.5^{\circ}$ ILAT bin is covered.

(2) The minimum could be caused by unfavourable solar illumination, MLT, $K_{p}$ or solar cycle phase during the times the satellite probed the $R=3.75$ region. These factors can be ruled out because the minimum is seen in all MLT, $K_{p}$ and season plots separately, and also because data at this altitude were gathered during many years. Most data for
$3.75 R_{E}$ come from 1999, which is a solar maximum year, thus one would expect an even higher occurrence frequency there because $K_{p}$ is, on the average, higher. The fact that $K_{p}$ is higher can be seen for example by comparing the second panels (the orbital coverages) of Fig. 10 at the $R=3.75$ bin. Furthermore, the minimum is found at slightly different altitude for different $K_{p}$, which speaks in favour of a geophysical effect.

\section{Summary}

To find at which altitude auroral potential structures close, we have for the first time studied auroral electric fields in the wide radial distance range $1.5-6 R_{E}$ using a large statistical 

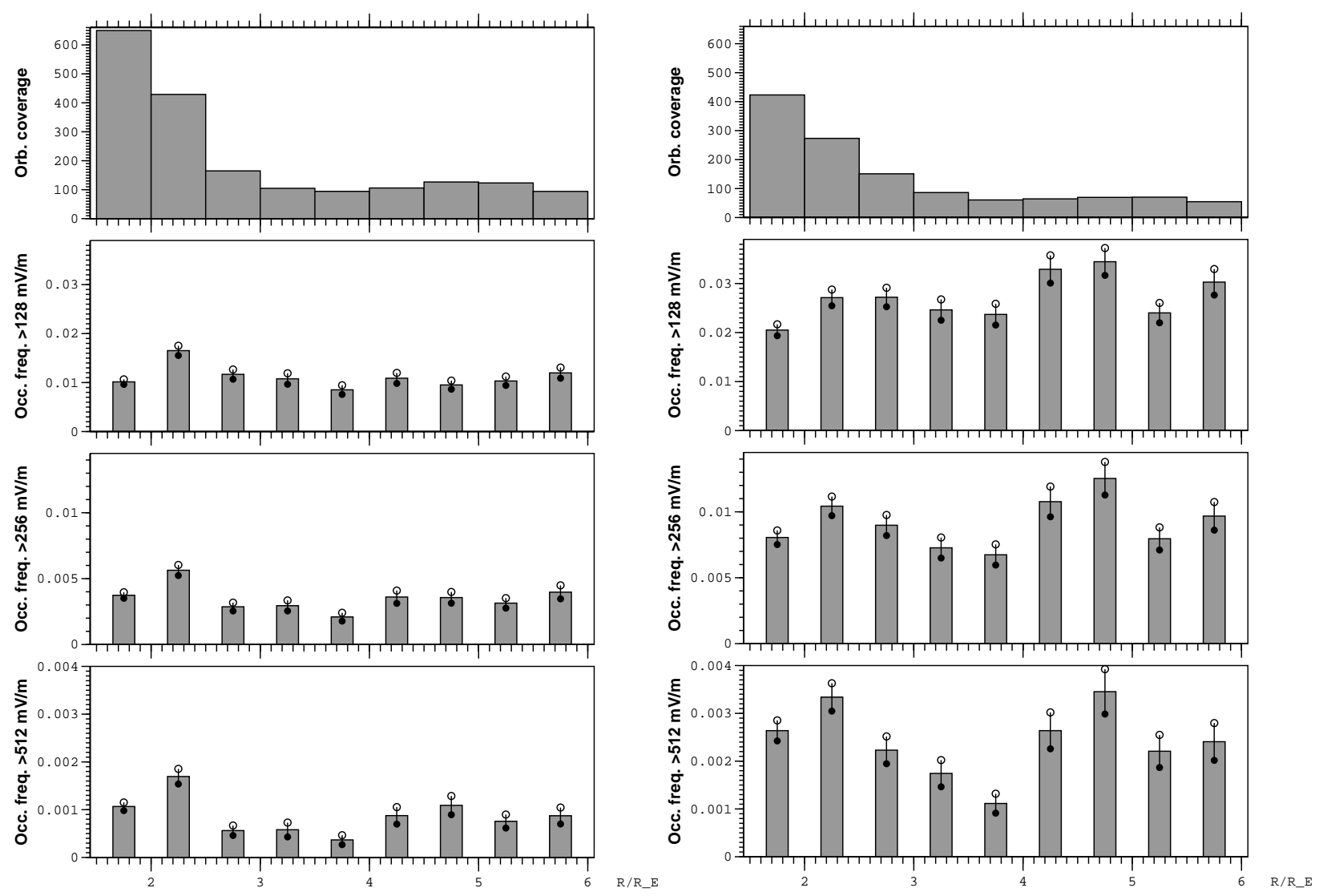

Fig. 14. Same as Fig. 11, but decomposed into small $K_{p}\left(K_{p} \leq 2\right.$, left $)$ and large $K_{p}\left(K_{p}>2\right.$, right).

database of the Polar satellite covering five years. From the analysis we conclude that there are two separate classes of electric field structures that are seen as potential minima by a satellite traversing them. The first class is the low-altitude potential minima which are associated with inverted-V electron spectra, auroral cavities and optical discrete arcs. These low-altitude potential minima are most often seen in the midnight and evening MLT sectors, and their altitude moves upward by $\sim 0.5-1 R_{E}$ when the ionospheric footpoint becomes Sun-illuminated. The strongest structures (in terms of their effective mapped-down ionospheric fields $E_{i}$ ) reside at the lowest altitudes, and the structures are about twice as common for $K_{p}>2$ than for $K_{p} \leq 2$.

Another class of electric field structures resides at high altitude $\left(R>4 R_{E}\right)$ in the midnight MLT sector. To some extent they also occur in the evening sector. This class of electric field structures is also a new finding of this paper, and their nature is still unknown to us. Since they occur predominantly in the midnight MLT sector and react to $K_{p}$ more strongly than the low-altitude fields, they are probably superposed substorm-related processes. They need not necessarily be quasi-static potential well structures in the same sense as the low-altitude ones, but could also correspond to temporally evolving structures (potential or inductive). Since the satellite ILAT speed is low at high altitudes, structures which oscillate in the north-south direction at a sufficiently rapid pace can encounter the satellite multiple times which may partly be the explanation for their relatively high occurrence frequency.

Equally interesting as the existence of the two classes of electric field structures is the relative rarity of the structures in middle altitudes $\left(R=3-4 R_{E}\right)$. In principle, concerning the potential structure closure question, two interpretations are possible:

1. The low-altitude potential structures do not extend beyond middle altitudes, which necessitates the existence of downward parallel electric fields in the intermediate region (Janhunen et al., 1999; Janhunen and Olsson, 2000, 2001, 2002; Hallinan and Stenbaek-Nielsen, 2001);

2. The low-altitude narrow structures widen before reaching higher altitudes so that the effective electric fields associated with them are reduced (Mozer and Hull, 2001).

The first possibility is called the Cooperative Model (for a recent review, see Olsson and Janhunen, 2003) and the latter possibility is called the potential finger model. In neither model can the structures above $4 R_{E}$ be related to the electrostatic potential structures of the auroral region. Figure 17 shows schematically the closed potential structure in the Cooperative Model for quiet arcs (top) and superposed 


\section{MLT 22-02, Darkness}

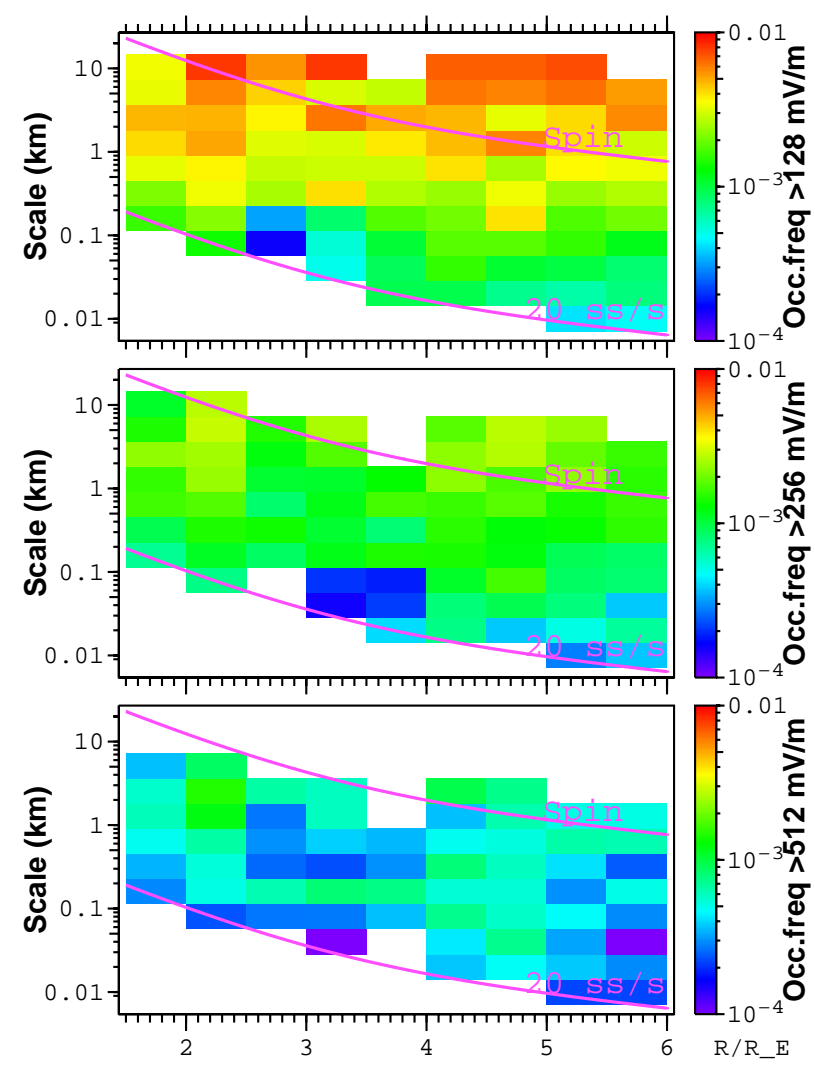

Fig. 15. Occurrence frequency of mapped-down effective electric field exceeding threshold $128 \mathrm{mV} / \mathrm{m}$ (top), $256 \mathrm{mV} / \mathrm{m}$ (second panel) and $512 \mathrm{mV} / \mathrm{m}$ (bottom), as a function of radial distance and mapped-down ionospheric scale size. Curves corresponding to satellite spin frequency $1 / 6 \mathrm{~Hz}$ and normal 20 samples per second are superposed. Data below the $20 \mathrm{~Hz}$ curve do not carry physical information and are thus shown as white.

with a substorm-time high-altitude electric field structure in the midnight MLT sector (bottom). The nature of the highaltitude fields is an open question. They could be electric fields of Alfvén waves or they could be quasi-electrostatic structures created at that altitude, for example by a Landau resonance between Alfvén waves and electrons.

To open up a new viewpoint to the data we also investigated the altitude statistics of all electric fields, not only those associated with potential minima. The results are similar to the potential structure results in all important ways. This suggests that the statistical results presented in this paper are not caused by problems in the minimum finding algorithm and that electric fields associated with potential structures are the most important class of electric fields found on auroral field lines.

A recent study of auroral density depletions shows that the depletions are concentrated mostly at low altitudes, however, with another island of high-altitude depletions appearing in the midnight MLT sector (Janhunen et al., 2002). This altitude pattern is very similar to the potential structure pat-
Orbital coverage, $R=2-3$

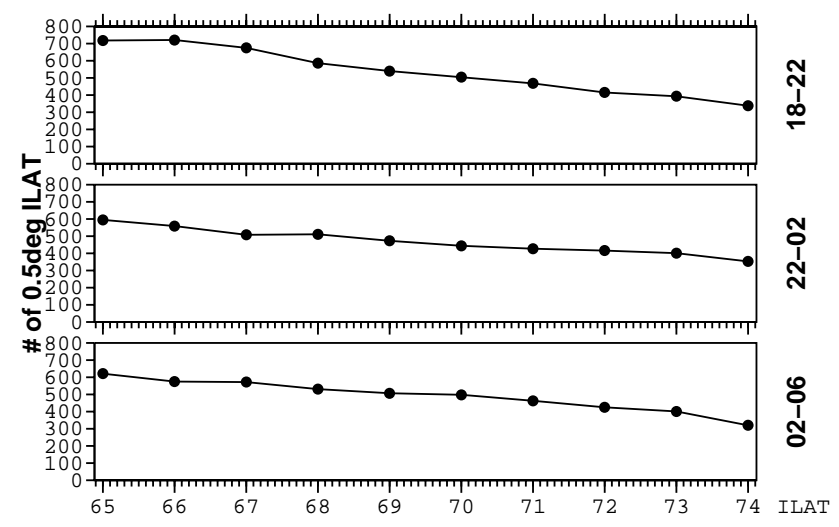

Orbital coverage, $R=3-4$

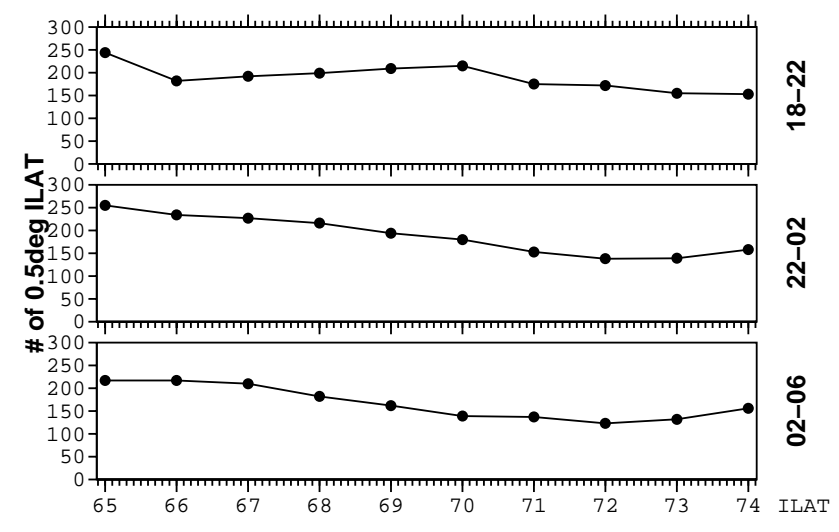

Orbital coverage, $R=4-5$

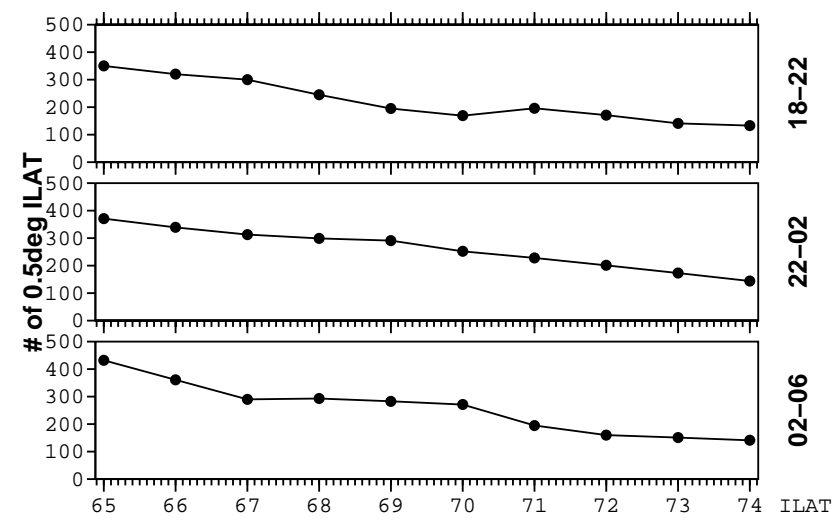

Fig. 16. Orbital coverage of Polar sectors satisfying our criteria of enough perpendicular trajectory in three radial distance ranges and in the three nightside MLT sectors.

tern found in the present paper. Also, it has been shown that the occurrence frequency of upgoing ion beams has a dip at 3.5-4 $R_{E}$ radial distance (Janhunen et al., 2003). Taken together, these results are consistent with the idea that the negative low-altitude potential structures correspond to density depletions. The working of the concept has also been demonstrated with a hybrid simulation (Janhunen and Olsson, 2002). 


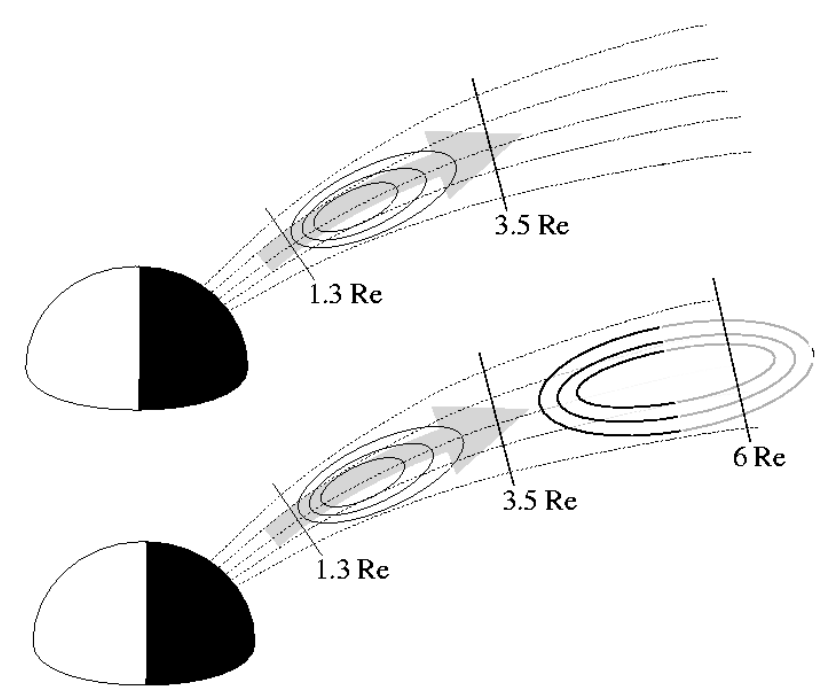

Fig. 17. Schematic figure containing the potential structure in the Cooperative Model for stable arcs (top), the same with a superposed high-altitude substorm-time electric field structure in the midnight MLT sector (bottom).

Acknowledgements. We are grateful to F. S. Mozer for providing the EFI data. We also thank C. T. Russell for providing MFE data. The work of PJ was supported by the Academy of Finland and that of AO by the Swedish Research Council. We are grateful to Andris Vaivads for useful comments of the manuscript.

The Editor in Chief thanks T. Hallinan and two other referees for their help in evaluating this paper.

\section{References}

Bennett, E. L., Temerin, M., and Mozer, F. S.: The distribution of auroral electrostatic shocks below 8000-km altitude, J. Geophys. Res., 88, 7107-7120, 1983.

Borovsky, J.: Auroral arc thicknesses as predicted by various theories, J. Geophys. Res., 98, 6101-6183, 1993.

Bryant, D. A. and Perry, C. H.: Velocity-space distributions of wave-accelerated auroral electrons, J. Geophys. Res., 100, 23 711-23 725, 1995.

Carlqvist, P. and Boström, R.: Space-charge regions above the aurora, J. Geophys. Res., 75, 7140-7146, 1970.

Haerendel, G., Buchert, S., LaHoz, C., Raaf, B., and Rieger, E.: On the proper motion of auroral arcs, J. Geophys. Res., 98, 60876099, 1993.

Hallinan, T. J., Kimball, J., Stenbaek-Nielsen, H. C., Lynch, K., Arnoldy, R., Bonnell, J., and Kintner, P.: Relation between optical emissions, particles, electric fields, and Alfvén waves in a multiple rayed arc, J. Geophys. Res., 106, 15 445-15 454, 2001.

Hallinan, T. J., and Stenbaek-Nielsen, H. C.: The connection between auroral acceleration and auroral morphology, Phys. Chem. Earth, 26, 169-177, 2001.

Harvey, P., Mozer, F. S., Pankow, D., Wygant, J., Maynard, N. C., Singer, H., Sullivan, W., Anderson, P. B., Pfaff, R., Aggson, T., Pedersen, A., Fälthammar, C. G., and Tanskanen, P.: The electric field instrument on the Polar satellite, Space Science Reviews, 71, 583-596, 1995.
Holzworth, R. H. and Meng, C.-I.: Mathematical representation of the auroral oval, Geophys. Res. Lett., 2, 377-380, 1975.

Janhunen, P., Olsson, A., Mozer, F. S ., and Laakso, H.: How does the U-shaped potential close above the acceleration region? A study using Polar data, Ann. Geophysicae, 17, 1276-1283, 1999.

Janhunen, P. and Olsson, A.: New model for auroral acceleration: O-shaped potential structure cooperating with waves, Ann. Geophysicae, 18, 596-607, 2000.

Janhunen, P. and Olsson, A.: Auroral potential structures and current-voltage relationship: summary of recent results, Phys. Chem. Earth, 26, 107-111, 2001.

Janhunen, P., and Olsson, A.: A hybrid simulation model for a stable auroral arc, Ann. Geophysicae, 20, 1603-1616, 2002.

Janhunen, P., Olsson, A., and Laakso, H.: Altitude dependence of plasma density in the auroral zone, Ann. Geophysicae, 20, 17431750, 2002.

Janhunen, P., Olsson, A., and Peterson W. K.: The occurrence frequency of upward ion beams in the auroral zone as a function of altitude using Polar/TIMAS and DE-1/EICS data, Ann. Geophysicae, 21, 2059-2072, 2003.

Knudsen, D. J., Donovan, E. F., Cogger, L. L., Jackel, B., and Shaw, W. D.: Width and structure of mesoscale optical auroral arcs Geophys. Res. Lett., 28, 705-708, 2001.

Lin, C. S. and Hoffman, R. A.: Observations of inverted-V electron precipitation, Space Sci. Rev., 30, 415-457, 1982.

Lindqvist, P.-A. and Marklund, G. T.: A statistical study of highaltitude electric fields measured on the Viking satellite, J. Geophys. Res., 95, 5867-5876, 1990.

Maggs, J. E. and Davis, T. N.: Measurements of the thicknesses of auroral structures, Planet. Space Sci., 16, 205, 1968.

McFadden, J. P., Carlson, C. W., Ergun, R. E., Mozer, F. S., Temerin, M., Peria, W., Klumpar, D. M., Shelley, E. G., Peterson, W. K., Moebius, E., Kistler, L. , Elphic, R. , Strangeway, R., Cattell, C., and Pfaff, R.: Spatial structure and gradients of ion beams observed by FAST, Geophys. Res. Lett., 25, 2021-2024, 1998.

Moore, T. E., Chappell, C. R., Chandler, M. O., Fields, S. A., Pollock, C. J., Reasoner, D. L., Young, D. T., Burch, J. L., Eaker, N., Waite Jr., J. H., McComas, D. J., Nordholt, J. E., Thomsen, M. F., Berthelier, J. J., and Robson, R.: The Thermal Ion Dynamics Experiment and Plasma Source Instrument, Space Sci. Revs., 71, 409, 1995.

Mozer, F. S., Carlson, C. W., Hudson, M. K., Torbert, R. B., Parady, B., Yatteau, I., and Kelley, M. C.: Observations of paired electrostatic shocks in the polar magnetosphere, Phys. Rev. Lett., 38, 292, 1977.

Mozer, F. S., Cattell, C. A., Hudson, M. K., Lysak, R. L., Temerin, M., and Torbert, R. B.: Satellite measurements and theories of low altitude auroral particle acceleration, Space Sci. Rev., 27, 155-213, 1980.

Mozer, F. S. and Hull, A.: Origin and geometry of upward parallel electric fields in the auroral acceleration region, J. Geophys. Res., 106, 5763-5778, 2001.

Newell, P. T., Meng, C.-I., and Lyons, K. M.: Suppression of discrete aurora by sunlight, Nature 381, 766-767, 1996.

Olsson, A. and Janhunen, P.: Some recent developments in understanding auroral electron acceleration processes, IEEE Trans. Plasma Sci., in press, 2004.

Russell, C. T., Snare, R. C., Means, J. D., Pierce, D., Dearborn, D., Larson, M., Barr, G., and Le, G.: The GGS/Polar Magnetic Fields Investigation, Space Sci. Rev., 71, 563-582, 1995. 
Stenbaek-Nielsen, H. C., Hallinan, T. J., Osborne, D. L., Kimball, J., Chaston, C., McFadden, J., Delory, G., Temerin, M., and Carlson, C. W.: Aircraft observations conjugate to FAST: auroral arc thickness, Geophys. Res. Lett., 25, 2073-2076, 1998.

Torbert, R. B. and Mozer, F. S.: Electrostatic shocks as the source of discrete auroral arcs, Geophys. Res. Lett., 5, 135-138, 1978.
Trondsen, T. S. and Cogger, L. L.: Fine-scale optical observations of aurora, Phys. Chem. Earth, 26, 179-188, 2001.

Williams, P. J. S., del Pozo, C. F., Hiscock, I., and Fallows, R.: Velocity of auroral arcs drifting equatorward from the polar cap, Ann. Geophys., 16, 1322-1331, 1998. 